\title{
Weighted norm inequality for bilinear Calderón-Zygmund operators on Herz-Morrey spaces with variable exponents
}

Shengrong Wang ${ }^{1}$ and Jingshi $\mathrm{Xu}^{2^{*}}$ (D)

"Correspondence:

jingshixu@126.com

${ }^{2}$ School of Mathematics and

Computing Science, Guilin

University of Electronic Technology,

Guilin, China

Full list of author information is

available at the end of the article

\begin{abstract}
In this paper, we obtain a weighted norm inequality of bilinear Calderón-Zygmund operators in Herz-Morrey spaces with variable exponents and weight in the variable Muckenhoupt class.

Keywords: Bilinear Calderón-Zygmund operator; Muckenhoupt weight; Variable exponent; Herz-Morrey space
\end{abstract}

\section{Introduction}

We denote by $\mathcal{S}\left(\mathbb{R}^{n}\right)$ the space of all Schwartz functions on $\mathbb{R}^{n}$ and by $\mathcal{S}^{\prime}\left(\mathbb{R}^{n}\right)$ the space of all tempered distributions on $\mathbb{R}^{n}$. Let $T$ be a bilinear operator, which is originally defined on the 2 -fold of Schwartz function space $\mathcal{S}\left(\mathbb{R}^{n}\right)$, and its value belongs to $\mathcal{S}^{\prime}\left(\mathbb{R}^{n}\right)$ :

$$
T: \mathcal{S}\left(\mathbb{R}^{n}\right) \times \mathcal{S}\left(\mathbb{R}^{n}\right) \rightarrow \mathcal{S}^{\prime}\left(\mathbb{R}^{n}\right) .
$$

$T$ is called bilinear Calderón-Zygmund operator, if it extends to a bounded bilinear operator from $L^{p_{1}} \times L^{p_{2}}$ to $L^{p}$ with $1 / p_{1}+1 / p_{2}=1 / p$, and for $f_{1}, f_{2} \in L_{C}^{\infty}\left(\mathbb{R}^{n}\right)$ (the space of compactly supported bounded functions), $x \notin \operatorname{supp}\left(f_{1}\right) \cap \operatorname{supp}\left(f_{2}\right)$

$$
T\left(f_{1}, f_{2}\right)(x):=\int_{\mathbb{R}^{2 n}} K\left(x, y_{1}, y_{2}\right) f_{1}\left(y_{1}\right) f_{2}\left(y_{2}\right) d y_{1} d y_{2},
$$

where the kernel $K$ is a function in $\mathbb{R}^{3 n}$ off from the diagonal $x=y_{1}=y_{2}$ and there exist positive constants $\varepsilon, A$ such that

$$
\left|K\left(x, y_{1}, y_{2}\right)\right| \leq \frac{A}{\left(\left|x-y_{1}\right|+\left|x-y_{2}\right|+\left|y_{1}-y_{2}\right|\right)^{2 n}}
$$

and

$$
\left|K\left(x, y_{1}, y_{2}\right)-K\left(x^{\prime}, y_{1}, y_{2}\right)\right| \leq \frac{A\left|x-x^{\prime}\right|^{\varepsilon}}{\left(\left|x-y_{1}\right|+\left|x-y_{2}\right|+\left|y_{1}-y_{2}\right|\right)^{2 n+\varepsilon}}
$$

whenever $\left|x-x^{\prime}\right| \leq \frac{1}{2} \max \left\{\left|x-y_{1}\right|,\left|x-y_{2}\right|\right\}$, and the two analogous difference estimates with respect to the variables $y_{1}$ and $y_{2}$ hold.

(c) The Author(s) 2019. This article is distributed under the terms of the Creative Commons Attribution 4.0 International License (http://creativecommons.org/licenses/by/4.0/), which permits unrestricted use, distribution, and reproduction in any medium, provided you give appropriate credit to the original author(s) and the source, provide a link to the Creative Commons license, and indicate if changes were made. 
Recently, Cruz-Uribe and Guzman proved the boundedness of the bilinear CalderónZygmund operator on products of weighted variable Lebesgue spaces in [1]. As a generalization of variable Lebesgue spaces, variable and weighted variable Herz-Morrey (Herz) spaces have been introduced in the last decades; see [2-11]. Motivated by [1], in this paper, we will prove a weighted norm inequality on products of Herz-Morrey spaces with variable exponents and weight in the variable Muckenhoupt class. We only consider the bilinear Calderón-Zygmund operator for simplicity. The analogs of our result for $m$-linear Calderón-Zygmund operators also hold for $m \geq 3$, because our argument and Lemma 8 in Sect. 3 also hold for $m$-linear Calderón-Zygmund operators with $m \geq 3$, see Remark 2.7 for [1, Theorem 2.4] in [1]. We mention here that the theory of multilinear CalderónZygmund operators started in [12]. After that, the boundedness of multilinear CalderónZygmund operators on products of various spaces has been obtained; see [13-19].

The plan of the paper is as follows. In Sect. 2, we collect some notations and state main result. The proof of the main result will be given in Sect. 3 .

\section{Notations and main result}

In this section, we firstly recall some definitions and notations, then we state our results. Let $\Omega$ be a positive measurable subset of $\mathbb{R}^{n}$, given a measurable function $p(\cdot): \Omega \rightarrow$ $[1, \infty)$, the Lebesgue space with variable exponent $L^{p(\cdot)}(\Omega)$ is defined by

$$
L^{p(\cdot)}(\Omega):=\left\{f \text { is measurable: } \int_{\mathbb{R}^{n}}\left(\frac{|f(x)|}{\lambda}\right)^{p(x)} d x<\infty \text { for some } \lambda>0\right\} .
$$

The Lebesgue space $L^{p(\cdot)}(\Omega)$ becomes a Banach function space equipped with the norm

$$
\|f\|_{L^{p(\cdot)}}:=\inf \left\{\lambda>0: \int_{\Omega}\left(\frac{|f(x)|}{\lambda}\right)^{p(x)} d x \leq 1\right\}
$$

The space $L_{\text {loc }}^{p(\cdot)}\left(\mathbb{R}^{n}\right)$ is defined by $L_{\text {loc }}^{p(\cdot)}\left(\mathbb{R}^{n}\right):=\left\{f: f \chi_{K} \in L^{p(\cdot)}\left(\mathbb{R}^{n}\right)\right.$ for all compact subsets $K \subset \mathbb{R}^{n}$, where and what follows, $\chi_{S}$ denotes the characteristic function of a measurable set $S \subset \mathbb{R}^{n}$. Let $p(\cdot): \mathbb{R}^{n} \rightarrow(0, \infty)$, we denote $p_{-}:=\operatorname{essinf}_{x \in \mathbb{R}^{n}} p(x), p_{+}:=\operatorname{ess~sup}_{x \in \mathbb{R}^{n}} p(x)$. The set $\mathcal{P}\left(\mathbb{R}^{n}\right)$ consists of all $p(\cdot)$ satisfying $p_{-}>1$ and $p_{+}<\infty ; \mathcal{P}_{0}\left(\mathbb{R}^{n}\right)$ consists of all $p(\cdot)$ satisfying $p_{-}>0$ and $p_{+}<\infty . L^{p(\cdot)}$ can be similarly defined as above for $p(\cdot) \in \mathcal{P}_{0}\left(\mathbb{R}^{n}\right) \cdot p^{\prime}(\cdot)$ means that the conjugate exponent of $p(\cdot)$, that means $1 / p(\cdot)+1 / p^{\prime}(\cdot)=1$.

Let $p(\cdot) \in \mathcal{P}\left(\mathbb{R}^{n}\right)$ and $w$ be a weight which is a non-negative measurable function on $\mathbb{R}^{n}$. Then the weighted variable exponent Lebesgue space $L^{p(\cdot)}(w)$ is the set of all complexvalued measurable function $f$ such that $f w \in L^{p(\cdot)}$. The space $L^{p(\cdot)}(w)$ is a Banach space equipped with the norm

$$
\|f\|_{L^{p(\cdot)(w)}}:=\|f w\|_{L^{p(\cdot)}}
$$

Let $f \in L_{\mathrm{loc}}^{1}\left(\mathbb{R}^{n}\right)$. Then the standard Hardy-Littlewood maximal function of $f$ is defined by

$$
M f(x):=\sup _{B \ni x} \frac{1}{|B|} \int_{B}|f(y)| d y, \quad \forall x \in \mathbb{R}^{n}
$$


where the supremum is taken over all balls containing $x$ in $\mathbb{R}^{n}$. In general, the HardyLittlewood maximal operator is not bounded on weighted variable Lebesgue spaces. But if $p(\cdot) \in \mathcal{P}\left(\mathbb{R}^{n}\right)$ and satisfies the following global log-Hölder continuous and $w \in A_{p(\cdot)}$, then $M$ is bounded on $L^{p(\cdot)}(w)$.

Definition 1 Let $\alpha(\cdot)$ be a real-valued measurable function on $\mathbb{R}^{n}$.

(i) The function $\alpha(\cdot)$ is locally log-Hölder continuous if there exists a constant $C_{1}$ such that

$$
|\alpha(x)-\alpha(y)| \leq \frac{C_{1}}{\log (e+1 /|x-y|)}, \quad x, y \in \mathbb{R}^{n},|x-y|<\frac{1}{2} .
$$

(ii) The function $\alpha(\cdot)$ is log-Hölder continuous at the origin if there exists a constant $C_{2}$ such that

$$
|\alpha(x)-\alpha(0)| \leq \frac{C_{2}}{\log (e+1 /|x|)}, \quad \forall x \in \mathbb{R}^{n} .
$$

Denote by $\mathcal{P}_{0}^{\log }\left(\mathbb{R}^{n}\right)$ the set of all log-Hölder continuous functions at the origin.

(iii) The function $\alpha(\cdot)$ is log-Hölder continuous at infinity if there exist $\alpha_{\infty} \in \mathbb{R}$ and a constant $C_{3}$ such that

$$
\left|\alpha(x)-\alpha_{\infty}\right| \leq \frac{C_{3}}{\log (e+|x|)}, \quad \forall x \in \mathbb{R}^{n} .
$$

Denote by $\mathcal{P}_{\infty}^{\log }\left(\mathbb{R}^{n}\right)$ the set of all log-Hölder continuous functions at infinity.

(iv) The function $\alpha(\cdot)$ is global log-Hölder continuous if $\alpha(\cdot)$ are both locally log-Hölder continuous and log-Hölder continuous at infinity. Denote by $\mathcal{P}^{\log }\left(\mathbb{R}^{n}\right)$ the set of all global log-Hölder continuous functions.

Definition 2 Let $p(\cdot) \in \mathcal{P}\left(\mathbb{R}^{n}\right)$, a positive measurable function $w$ is said to be in $A_{p(\cdot)}$, if exists a positive constant $C$ for all balls $B$ in $\mathbb{R}^{n}$ such that

$$
\sup _{B} \frac{1}{|B|}\left\|w \chi_{B}\right\|_{L^{p(\cdot)}}\left\|w^{-1} \chi_{B}\right\|_{L^{p^{(} \cdot()}}<\infty .
$$

Remark 1 In [20], Cruz-Uribe, Fiorenza and Neugebauer found that if $p(\cdot) \in \mathcal{P}\left(\mathbb{R}^{n}\right)$ and $w \in A_{p(\cdot)}$, then $w^{-1} \in A_{p^{\prime}(\cdot)}$.

The Muckenhoupt $A_{p}$ class with constant exponent $p \in(1, \infty)$ firstly proposed by Muckenhoupt in [21]. The variable Muckenhoupt $A_{p(.)}$ was considered in [20, 22-25].

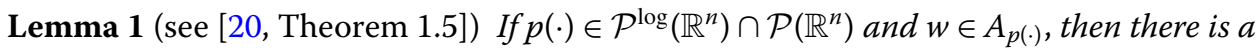
positive constant $C$ such that, for each $f \in L^{p(\cdot)}(w)$,

$$
\|(M f) w\|_{L^{p(\cdot)}} \leq C\|f w\|_{L^{p^{(\cdot)}}} .
$$

To give the definitions of the Herz space and the Herz-Morrey space with variable exponents, we use the following notations. For each $k \in \mathbb{Z}$ we define

$$
B_{k}:=\left\{x \in \mathbb{R}^{n}:|x| \leq 2^{k}\right\}, \quad D_{k}:=B_{k} \backslash B_{k-1},
$$




$$
\chi_{k}:=\chi_{D_{k}}, \quad \tilde{\chi}_{m}=\chi_{m}, \quad m \geq 1, \tilde{\chi}_{0}=\chi_{B_{0}} .
$$

Definition 3 Let $q \in(0, \infty], p(\cdot) \in \mathcal{P}_{0}\left(\mathbb{R}^{n}\right)$, and $\alpha(\cdot): \mathbb{R}^{n} \rightarrow \mathbb{R}$ with $\alpha \in L^{\infty}\left(\mathbb{R}^{n}\right)$.

(1) The homogeneous weighted Herz space $\dot{K}_{p(\cdot)}^{\alpha(\cdot), q}(w)$ is defined by

$$
\dot{K}_{p(\cdot)}^{\alpha(\cdot), q}(w):=\left\{f \in L_{\text {loc }}^{p(\cdot)}\left(\mathbb{R}^{n} \backslash\{0\}, w\right):\|f\|_{\dot{K}_{p(\cdot)}^{\alpha(\cdot), q(w)}}<\infty\right\}
$$

where

$$
\|f\|_{\dot{K}_{p(\cdot)}^{\alpha(\cdot), q(w)}}:=\left\{\sum_{k=-\infty}^{\infty}\left\|2^{k \alpha(\cdot)} f \chi_{k}\right\|_{L^{p(\cdot)}(w)}^{q}\right\}^{1 / q} .
$$

(2) The inhomogeneous weighted Herz space $K_{p(\cdot)}^{\alpha(\cdot), q}(w)$ is defined by

$$
K_{p(\cdot)}^{\alpha(\cdot), q}(w):=\left\{f \in L_{\mathrm{loc}}^{p(\cdot)}(w):\|f\|_{K_{p(\cdot)}^{\alpha(\cdot), q(w)}}<\infty\right\}
$$

where

$$
\|f\|_{K_{p(\cdot)}^{\alpha(\cdot), q(w)}}:=\left\{\sum_{m=0}^{\infty}\left\|2^{m \alpha(\cdot)} f \widetilde{\chi}_{m}\right\|_{L^{p(\cdot)(w)}}^{q}\right\}^{1 / q} .
$$

Remark 2 If $0<q_{1} \leq q_{2} \leq \infty$ and $w \equiv 1$, then $\dot{K}_{p(\cdot)}^{\alpha(\cdot), q_{1}}\left(\mathbb{R}^{n}\right) \subset \dot{K}_{p(\cdot)}^{\alpha(\cdot), q_{2}}\left(\mathbb{R}^{n}\right)$. If $w \equiv 1, \alpha(\cdot)$ and $p(\cdot)$ are constants, then $\dot{K}_{p(\cdot)}^{\alpha(\cdot), q}\left(\mathbb{R}^{n}\right)=\dot{K}_{p}^{\alpha, q}\left(\mathbb{R}^{n}\right)$ is the classical Herz spaces in [26, 27].

To generalize the above spaces to variable exponent $q(\cdot)$, we need the notation of the variable mixed sequence space $\ell^{q(\cdot)}\left(L^{p(\cdot)}\right)$, which is firstly defined by Almeida and Hästö in [28]. Let $w$ be a non-negative measurable function. Given a sequence of functions $\left\{f_{j}\right\}_{j \in \mathbb{Z}}$, define the modular

$$
\rho_{\ell q(\cdot)\left(L^{p(\cdot)}(w)\right)}\left(\left(f_{j}\right)_{j}\right):=\sum_{j \in \mathbb{Z}} \inf \left\{\lambda_{j}: \int_{\mathbb{R}^{n}}\left(\frac{\left|f_{j}(x) w(x)\right|}{\lambda_{j}^{\frac{1}{q(x)}}}\right)^{p(x)} d x \leq 1\right\},
$$

where $\lambda^{1 / \infty}=1$. If $q^{+}<\infty$ or $q(\cdot) \leq p(\cdot)$, the above can be written as

$$
\rho_{\ell q(\cdot)\left(L^{p \cdot \cdot(\cdot))}\right.}\left(\left(f_{j}\right)_{j}\right)=\sum_{j \in \mathbb{Z}}\left\|\left|f_{j} w\right|^{q(\cdot)}\right\|_{L^{\frac{p(\cdot)}{q \cdot)}}}
$$

The norm is

$$
\left\|\left(f_{j}\right)_{j}\right\|_{\ell q(\cdot)\left(L^{p(\cdot)(w))}\right.}:=\inf \left\{\mu>0: \rho_{\ell q(\cdot)\left(L^{p \cdot \cdot(w))}\right.}\left(\left(f_{j} / \mu\right)_{j}\right) \leq 1\right\} .
$$

Now, spaces $\dot{K}_{p(\cdot)}^{\alpha(\cdot), q(\cdot)}(w)$ and $K_{p(\cdot)}^{\alpha(\cdot), q(\cdot)}(w)$ are defined, respectively, by

$$
\dot{K}_{p(\cdot)}^{\alpha(\cdot), q(\cdot)}(w):=\left\{f \in L_{\mathrm{loc}}^{p(\cdot)}\left(\mathbb{R}^{n} \backslash\{0\}, w\right):\|f\|_{\dot{K}_{p(\cdot)}^{\alpha(\cdot),(\cdot)}(w)}<\infty\right\},
$$


where

$$
\|f\|_{\dot{K}_{p(\cdot)}^{\alpha(\cdot), q(\cdot)}(w)}:=\left\|\left(2^{j \alpha(\cdot)} f \chi_{j}\right)_{j}\right\|_{\ell q(\cdot)\left(L^{p(\cdot)}(w)\right)}
$$

and

$$
K_{p(\cdot)}^{\alpha(\cdot), q(\cdot)}(w):=\left\{f \in L_{\mathrm{loc}}^{p(\cdot)}(w):\|f\|_{K_{p(\cdot)}^{\alpha(\cdot), q(\cdot)}(w)}=\left\|\left(2^{j \alpha(\cdot)} f \chi_{j}\right)_{j}\right\|_{\ell q(\cdot)\left(L^{p(\cdot)}(w)\right)}<\infty\right\} .
$$

For any quantities $A$ and $B$, we shall write $A \lesssim B$ to indicate that there exists a constant $C>0$ such that $A \leq C B$. If $A \lesssim B$ and $B \lesssim A$, we write $A \approx B$.

The following lemma is a corollary of [29, Theorem 3].

Lemma 2 Let $\alpha(\cdot) \in L^{\infty}\left(\mathbb{R}^{n}\right), p(\cdot), q(\cdot) \in \mathcal{P}_{0}\left(\mathbb{R}^{n}\right)$ and $w$ be a weight. If $\alpha(\cdot)$ and $q(\cdot)$ are log-Hölder continuous at infinity, then

$$
K_{p(\cdot)}^{\alpha(\cdot), q(\cdot)}(w)=K_{p(\cdot)}^{\alpha \infty, q_{\infty}}(w)
$$

Additionally, if $\alpha(\cdot)$ and $q(\cdot)$ are log-Hölder continuous at the origin, then

$$
\begin{aligned}
\|f\|_{\dot{K}_{p(\cdot)}^{\alpha(\cdot), q(\cdot)}(w)} \approx & \left(\sum_{k \leq 0}\left\|2^{k \alpha(0)} f \chi_{k}\right\|_{L^{p \cdot(\cdot)}(w)}^{q(0)}\right)^{1 / q(0)} \\
& +\left(\sum_{k>0}\left\|2^{k \alpha \infty} f \chi_{k}\right\|_{L^{p(\cdot)}(w)}^{q_{\infty}}\right)^{1 / q_{\infty}} .
\end{aligned}
$$

Definition 4 Let $p(\cdot), q(\cdot) \in \mathcal{P}_{0}\left(\mathbb{R}^{n}\right), \lambda \in[0, \infty)$. Let $\alpha(\cdot)$ be a bounded real-valued measurable function on $\mathbb{R}^{n}$. The homogeneous weighted Herz-Morrey space $M \dot{K}_{p(\cdot), \lambda}^{\alpha(\cdot), q(\cdot)}(w)$ and non-homogeneous weighted Herz-Morrey space $M K_{p(\cdot), \lambda}^{\alpha(\cdot),(\cdot)}(w)$ are defined, respectively, by

$$
M \dot{K}_{p(\cdot), \lambda}^{\alpha(\cdot), q(\cdot)}(w):=\left\{f \in L_{\text {loc }}^{p(\cdot)}\left(\mathbb{R}^{n} \backslash\{0\}, w\right):\|f\|_{M \dot{K}_{p(\cdot), \lambda}^{\alpha(\cdot),(\cdot)}(w)}<\infty\right\}
$$

and

$$
M K_{p(\cdot), \lambda}^{\alpha(\cdot), q(\cdot)}(w):=\left\{f \in L_{\mathrm{loc}}^{p(\cdot)}\left(\mathbb{R}^{n}, w\right):\|f\|_{M K_{p(\cdot), \lambda}^{\alpha(\cdot),(\cdot)}(w)}<\infty\right\},
$$

where

$$
\|f\|_{M \dot{K}_{p(\cdot), \lambda}^{\alpha(\cdot), q(\cdot)}(w)}:=\sup _{L \in \mathbb{Z}} 2^{-L \lambda}\left\|\left(2^{\alpha(\cdot) k} f \chi_{k}\right)_{k \leq L}\right\|_{\ell q(\cdot)\left(L^{(\cdot)}(w)\right)}
$$

and

$$
\|f\|_{M K_{p(\cdot), \lambda}^{\alpha(\cdot),(\cdot)}(w)}:=\sup _{L \in \mathbb{N}_{0}} 2^{-L \lambda}\left\|\left(2^{\alpha(\cdot) k} f \tilde{\chi}_{k}\right)_{k=0}^{L}\right\|_{\ell q(\cdot)\left(L^{p(\cdot)}(w)\right)} .
$$

Proposition 1 Let $p(\cdot), q(\cdot) \in \mathcal{P}_{0}\left(\mathbb{R}^{n}\right)$, w be a weight, $\lambda \in[0, \infty)$, and $\alpha(\cdot) \in L^{\infty}\left(\mathbb{R}^{n}\right)$. 
(i) If $\alpha(\cdot), q(\cdot) \in \mathcal{P}_{0}^{\log }\left(\mathbb{R}^{n}\right) \cap \mathcal{P}_{\infty}^{\log }\left(\mathbb{R}^{n}\right)$, then, for any $f \in L_{\text {loc }}^{p(\cdot)}\left(\mathbb{R}^{n} \backslash\{0\}, w\right)$,

$$
\begin{aligned}
& \|f\|_{M \dot{K}_{p(\cdot), \lambda}^{\alpha(\cdot),(\cdot)}(w)} \\
& \approx \max \left\{\sup _{L \leq 0, L \in \mathbb{Z}} 2^{-L \lambda}\left\|\left(2^{k \alpha(0)} f \chi_{k}\right)_{k \leq L}\right\|_{\ell^{q_{0}\left(L^{p(\cdot)}(w)\right)},}\right. \\
& \left.\quad \sup _{L>0, L \in \mathbb{Z}}\left[2^{-L \lambda}\left\|\left(2^{k \alpha(0)} f \chi_{k}\right)_{k<0}\right\|_{\ell^{q_{0}\left(L^{p(\cdot)}(w)\right)}}+2^{-L \lambda}\left\|\left(2^{k \alpha_{\infty}} f \chi_{k}\right)_{k=0}^{L}\right\|_{\ell q \infty\left(L^{p(\cdot)}(w)\right)}\right]\right\},
\end{aligned}
$$

where throughout $q_{0}:=q(0)$.

(ii) If $\alpha(\cdot), q(\cdot) \in \mathcal{P}_{\infty}^{\log }\left(\mathbb{R}^{n}\right)$, then

$$
M K_{p(\cdot), \lambda}^{\alpha(\cdot), q(\cdot)}(w)=M K_{p(\cdot), \lambda}^{\alpha_{\infty}, q_{\infty}}(w)
$$

Proof Obviously,

$$
\begin{aligned}
\|f\|_{M \dot{K}_{p(\cdot), \lambda}^{\alpha(\cdot), q(\cdot)}(w)}= & \max \left\{\sup _{L \leq 0, L \in \mathbb{Z}} 2^{-L \lambda}\left\|\left(2^{k \alpha(\cdot)} f \chi_{k}\right)_{k \leq L}\right\|_{\ell q(\cdot)\left(L^{p(\cdot)}(w)\right)},\right. \\
& \left.\sup _{L>0, L \in \mathbb{Z}} 2^{-L \lambda}\left\|\left(2^{k \alpha(\cdot)} f \chi_{k}\right)_{k \leq L}\right\|_{\ell q(\cdot)\left(L^{p(\cdot)(w)}\right)}\right\} .
\end{aligned}
$$

When $L \leq 0$, from Lemma 2 we know that

$$
\left\|\left(2^{k \alpha(\cdot)} f \chi_{k}\right)_{k \leq L}\right\|_{\ell q(\cdot)\left(L^{p(\cdot)}(w)\right)} \approx\left\|\left(2^{k \alpha(0)} f \chi_{k}\right)_{k \leq L}\right\|_{\ell q_{0}\left(L^{p(\cdot)}(w)\right)} .
$$

When $L>0$, from Lemma 2 again we also obtain

$$
\begin{aligned}
\left\|\left(2^{k \alpha(\cdot)} f \chi_{k}\right)_{k<L}\right\|_{\ell q(\cdot)\left(L^{p(\cdot)}(w)\right)} \approx & \left\|\left(2^{k \alpha(0)} f \chi_{k}\right)_{k<0}\right\|_{\ell q_{0}\left(L^{p \cdot()}(w)\right)} \\
& +\left\|\left(2^{k \alpha_{\infty}} f \chi_{k}\right)_{k=0}^{L}\right\|_{\ell q \infty\left(L^{p(\cdot)}(w)\right)} .
\end{aligned}
$$

Thus we obtain (i). Similarly, we obtain (ii).

Lemmas 3 and 4 below have been proved by Izuki and Noi in [30, 31].

Lemma 3 If $p(\cdot) \in \mathcal{P}^{\log }\left(\mathbb{R}^{n}\right) \cap \mathcal{P}\left(\mathbb{R}^{n}\right)$ and $w \in A_{p(\cdot)}$, then there exists a constant $C>0$ such that, for all balls $B$ in $\mathbb{R}^{n}$ and all measurable subsets $S \subset B$,

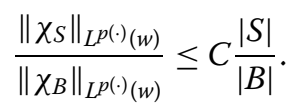

Lemma 4 If $p(\cdot) \in \mathcal{P}^{\log }\left(\mathbb{R}^{n}\right) \cap \mathcal{P}\left(\mathbb{R}^{n}\right)$ and $w \in A_{p(\cdot)}$, then there exist constants $\delta_{1}, \delta_{2} \in(0,1)$ and $C>0$ such that, for all balls $B$ in $\mathbb{R}^{n}$ and all measurable subsets $S \subset B$,

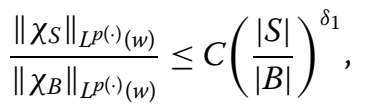

$$
\begin{aligned}
& \frac{\left\|\chi_{S}\right\|_{L^{p^{\prime}(\cdot)}\left(w^{-1}\right)}}{\left\|\chi_{B}\right\|_{L^{p^{\prime}(\cdot)}\left(w^{-1}\right)}} \leq C\left(\frac{|S|}{|B|}\right)^{\delta_{2}} \text {. }
\end{aligned}
$$




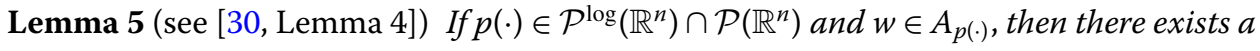
positive constant $C$ such that, for all balls $B$ in $\mathbb{R}^{n}$,

$$
C^{-1} \leq \frac{1}{|B|}\left\|\chi_{B}\right\|_{L^{p(\cdot)(w)}}\left\|\chi_{B}\right\|_{L^{p^{\prime}(\cdot)\left(w^{-1}\right)}} \leq C .
$$

Our main result is as follows.

Theorem 1 Assume that $T$ is a bilinear Calderón-Zygmund operator, $p_{1}(\cdot), p_{2}(\cdot) \in$ $\mathcal{P}^{\log }\left(\mathbb{R}^{n}\right) \cap \mathcal{P}\left(\mathbb{R}^{n}\right)$ satisfying $1 / p(x)=1 / p_{1}(x)+1 / p_{2}(x)$ for $x \in \mathbb{R}^{n}$. Let $w_{1}$, w $w_{2}$ be weights, $w=w_{1} w_{2}, w_{i} \in A_{p_{i}(\cdot),}, i=1,2$. Suppose that $\alpha(\cdot) \in L^{\infty}\left(\mathbb{R}^{n}\right) \cap \mathcal{P}_{0}^{\log }\left(\mathbb{R}^{n}\right) \cap \mathcal{P}_{\infty}^{\log }\left(\mathbb{R}^{n}\right), \alpha(0)=$ $\alpha_{1}(0)+\alpha_{2}(0), \alpha_{\infty}=\alpha_{1 \infty}+\alpha_{2 \infty}, q(\cdot) \in \mathcal{P}_{0}^{\log }\left(\mathbb{R}^{n}\right) \cap \mathcal{P}_{\infty}^{\log }\left(\mathbb{R}^{n}\right), 1 / q(0)=1 / q_{1}(0)+1 / q_{2}(0)$, $1 / q_{\infty}=1 / q_{1 \infty}+1 / q_{2 \infty}, \lambda=\lambda_{1}+\lambda_{2}, 0 \leq \lambda_{i}<\infty, \delta_{i 1}, \delta_{i 2} \in(0,1)$ are the constants in Lemma 4 for exponents $p_{i}(\cdot)$ and weights $w_{i}, i=1$, 2. If $\lambda_{i}+n \delta_{i 2}>\alpha_{i \infty} \geq \alpha_{i}(0)$ for $i=1,2$, then

$$
\left\|T\left(f_{1}, f_{2}\right)\right\|_{M \dot{K}_{p(\cdot), \lambda}^{\alpha(\cdot), q(\cdot)(w)}} \lesssim\left\|f_{1}\right\|_{M \dot{K}_{p_{1}(\cdot), \lambda_{1}}^{\alpha_{1}(\cdot), q_{1}(\cdot)}\left(w_{1}\right)}\left\|f_{2}\right\|_{M \dot{K}_{p_{2}(\cdot), \lambda_{2}}^{\alpha_{2}(\cdot), q_{2}(\cdot)}\left(w_{2}\right)} .
$$

From Theorem 1, we obtain the following corollary.

Corollary 1 Assume that $T$ is a bilinear Calderón-Zygmund operator, $p_{1}(\cdot), p_{2}(\cdot) \in$ $\mathcal{P}^{\log }\left(\mathbb{R}^{n}\right) \cap \mathcal{P}\left(\mathbb{R}^{n}\right)$ satisfying $1 / p(x)=1 / p_{1}(x)+1 / p_{2}(x)$ for $x \in \mathbb{R}^{n}$. Let $w_{1}$, w $w_{2}$ be weights, $w=w_{1} w_{2}, w_{i} \in A_{p_{i}(\cdot),}, i=1,2$. Suppose that $\alpha(\cdot) \in L^{\infty}\left(\mathbb{R}^{n}\right) \cap \mathcal{P}_{0}^{\log }\left(\mathbb{R}^{n}\right) \cap \mathcal{P}_{\infty}^{\log }\left(\mathbb{R}^{n}\right), \alpha(0)=$ $\alpha_{1}(0)+\alpha_{2}(0), \alpha_{\infty}=\alpha_{1 \infty}+\alpha_{2 \infty}, q(\cdot) \in \mathcal{P}_{0}^{\log }\left(\mathbb{R}^{n}\right) \cap \mathcal{P}_{\infty}^{\log }\left(\mathbb{R}^{n}\right), 1 / q(0)=1 / q_{1}(0)+1 / q_{2}(0)$, $1 / q_{\infty}=1 / q_{1 \infty}+1 / q_{2 \infty}, \delta_{i 1}, \delta_{i 2} \in(0,1)$ are the constants in Lemma 4 for exponents $p_{i}(\cdot)$ and weights $w_{i}, i=1$, 2. If $\lambda_{i}+n \delta_{i 2}>\alpha_{i \infty} \geq \alpha_{i}(0)$ for $i=1,2$, then

$$
\left\|T\left(f_{1}, f_{2}\right)\right\|_{\dot{K}_{p(\cdot)}^{\alpha(\cdot), q(\cdot)}(w)} \lesssim\left\|f_{1}\right\|_{\dot{K}_{p_{1}(\cdot)}^{\alpha_{1}(\cdot), q_{1}(\cdot)}}\left\|f_{\left(w_{1}\right)}\right\|_{\dot{K}_{p_{2}(\cdot)}^{\alpha_{2}(\cdot), q_{2}(\cdot)}\left(w_{2}\right)} \cdot
$$

\section{Proof of Theorem 1}

To prove Theorem 1, we need a series of lemmas.

Lemma 6 (see [16, Theorem 2.3]) Let $p(\cdot), p_{1}(\cdot), p_{2}(\cdot) \in \mathcal{P}_{0}\left(\mathbb{R}^{n}\right)$ such that $1 / p(x)=1 / p_{1}(x)+$ $1 / p_{2}(x)$ for $x \in \mathbb{R}^{n}$. Then there exists a constant $C_{p, p_{1}}$ independent of functions $f$ and $g$ such that

$$
\|f g\|_{L^{p(\cdot)}} \leq C_{p, p_{1}}\|f\|_{L^{p_{1}(\cdot)}}\|g\|_{L^{p_{2}(\cdot)}}
$$

holds for every $f \in L^{p_{1}(\cdot)}\left(\mathbb{R}^{n}\right)$ and $g \in L^{p_{2}(\cdot)}\left(\mathbb{R}^{n}\right)$. If $p \in \mathcal{P}\left(\mathbb{R}^{n}\right)$, w be weight with $w=w_{1} \times w_{2}$, then

$$
\|f g\|_{L^{p(\cdot)}(w)} \leq C_{p, p_{1}}\|f\|_{L^{p_{1}(\cdot)\left(w_{1}\right)}}\|g\|_{L^{p_{2}(\cdot)}\left(w_{2}\right)} \cdot
$$

Lemma 7 (see [32, Proposition 1.2]) Let $0<p \leq \infty, \delta>0$. Then there is a positive constant C such that

$$
\left(\sum_{j=-\infty}^{\infty}\left(\sum_{k=-\infty}^{\infty} 2^{-|k-j| \delta} a_{k}\right)^{p}\right)^{1 / p} \leq C\left(\sum_{j=-\infty}^{\infty} a_{j}^{p}\right)^{1 / p}
$$


for non-negative sequences $\left\{a_{j}\right\}_{j=-\infty}^{\infty}$. Here, when $p=\infty$, it is understood that (4) stands for

$$
\sup _{j \in \mathbb{Z}}\left(\sum_{k=-\infty}^{\infty} 2^{-|k-j| \delta} a_{k}\right) \leq C \sup _{j \in \mathbb{Z}} a_{j} .
$$

The following lemma is a corollary of [1, Theorem 2.8].

Lemma 8 Let $p_{1}(\cdot), p_{1}(\cdot) \in \mathcal{P}\left(\mathbb{R}^{n}\right), 1<\left(p_{i}\right)_{-} \leq\left(p_{i}\right)_{+}<\infty$ and $p_{i}(\cdot) \in \mathcal{P}^{\log }\left(\mathbb{R}^{n}\right) \cap \mathcal{P}\left(\mathbb{R}^{n}\right)$ satisfying $1 / p(x)=1 / p_{1}(x)+1 / p_{2}(x)$ for $x \in \mathbb{R}^{n}, i=1,2$. Let $w_{1} \in A_{p_{1}(\cdot)}, w_{2} \in A_{p_{2}(\cdot)}$ and $w=w_{1} w_{2}$. If $T$ is a bilinear Calderón-Zygmund operator, then

$$
\left\|T\left(f_{1}, f_{2}\right)\right\|_{L^{p \cdot()(w)}} \lesssim\left\|f_{1}\right\|_{L^{p_{1}(\cdot)}\left(w_{1}\right)}\left\|f_{2}\right\|_{L^{p_{2}(\cdot)}\left(w_{2}\right)} \cdot
$$

Proof of Theorem 1 Let $f_{1}$ and $f_{2}$ be bounded functions with compact support and write

$$
f_{i}=\sum_{l=-\infty}^{\infty} f_{i} \chi_{l}=: \sum_{l=-\infty}^{\infty} f_{i l}, \quad i=1,2 .
$$

By Proposition 1, we have

$$
\begin{aligned}
\left\|T\left(f_{1}, f_{2}\right)\right\|_{M \dot{K}_{p(\cdot), \lambda}^{\alpha(\cdot),(\cdot)}(w)} & \\
\approx & \max \left\{\sup _{L \leq 0, L \in \mathbb{Z}} 2^{-L \lambda}\left\|\left(2^{k \alpha(0)} T\left(f_{1}, f_{2}\right) \chi_{k}\right)_{k \leq L}\right\|_{l^{q_{0}\left(L^{p(\cdot)}(w)\right)},},\right. \\
& \sup _{L>0, L \in \mathbb{Z}}\left[2^{-L \lambda}\left\|\left(2^{k \alpha(0)} T\left(f_{1}, f_{2}\right) \chi_{k}\right)_{k<0}\right\|_{l^{q_{0}\left(L^{p(\cdot)}(w)\right)}}\right. \\
& \left.\left.+2^{-L \lambda}\left\|\left(2^{k \alpha \infty} T\left(f_{1}, f_{2}\right) \chi_{k}\right)_{k=0}^{L}\right\|_{l q^{\infty}\left(L^{p(\cdot)}(w)\right)}\right]\right\} \\
:= & \max \{E, F+G\},
\end{aligned}
$$

where

$$
\begin{aligned}
& E:=\sup _{L \leq 0, L \in \mathbb{Z}} 2^{-L \lambda}\left\|\left(2^{k \alpha(0)} T\left(f_{1}, f_{2}\right) \chi_{k}\right)_{k \leq L}\right\|_{l^{q_{0}\left(L^{p \cdot(\cdot)}(w)\right)},} \\
& F:=\sup _{L>0, L \in \mathbb{Z}} 2^{-L \lambda}\left\|\left(2^{k \alpha(0)} T\left(f_{1}, f_{2}\right) \chi_{k}\right)_{k<0}\right\|_{l^{q_{0}\left(L^{p \cdot(\cdot)}(w)\right)},}, \\
& G:=\sup _{L>0, L \in \mathbb{Z}} 2^{-L \lambda}\left\|\left(2^{k \alpha_{\infty}} T\left(f_{1}, f_{2}\right) \chi_{k}\right)_{k=0}^{L}\right\|_{l^{q_{\infty}\left(L^{p \cdot()}(w)\right)} \cdot},
\end{aligned}
$$

Since to estimate $F$ is essentially similar to estimate $E$, so it suffices for us to show that $E$ and $G$ are bounded in weighted Herz-Morrey space with variable exponents. It is easy to see that

$$
E \leq C \sum_{i=i}^{9} E_{i}, \quad G \leq C \sum_{i=i}^{9} G_{i},
$$

where

$$
E_{1}:=\sup _{L \leq 0, L \in \mathbb{Z}} 2^{-L \lambda}\left(\sum_{k=-\infty}^{L} 2^{k \alpha(0) q(0)}\left\|\sum_{l=-\infty}^{k-2} \sum_{j=-\infty}^{k-2} T\left(f_{1 l}, f_{2 j}\right) \chi_{k}\right\|_{L^{p(\cdot)(w)}}^{q(0)}\right)^{\frac{1}{q(0)}},
$$




$$
\begin{aligned}
& E_{2}:=\sup _{L \leq 0, L \in \mathbb{Z}} 2^{-L \lambda}\left(\sum_{k=-\infty}^{L} 2^{k \alpha(0) q(0)}\left\|\sum_{l=-\infty}^{k-2} \sum_{j=k-1}^{k+1} T\left(f_{1 l}, f_{2 j}\right) \chi_{k}\right\|_{L^{p(\cdot)}(w)}^{q(0)}\right)^{\frac{1}{q(0)}} \\
& E_{3}:=\sup _{L \leq 0, L \in \mathbb{Z}} 2^{-L \lambda}\left(\sum_{k=-\infty}^{L} 2^{k \alpha(0) q(0)}\left\|\sum_{l=-\infty}^{k-2} \sum_{j=k+2}^{\infty} T\left(f_{1 l}, f_{2 j}\right) \chi_{k}\right\|_{L^{p(\cdot)}(w)}^{q(0)}\right)^{\frac{1}{q(0)}} \\
& E_{4}:=\sup _{L \leq 0, L \in \mathbb{Z}} 2^{-L \lambda}\left(\sum_{k=-\infty}^{L} 2^{k \alpha(0) q(0)}\left\|\sum_{l=k-1}^{k+1} \sum_{j=-\infty}^{k-2} T\left(f_{1 l}, f_{2 j}\right) \chi_{k}\right\|_{L^{p(\cdot)}(w)}^{q(0)}\right)^{\frac{1}{q(0)}} \\
& E_{5}:=\sup _{L \leq 0, L \in \mathbb{Z}} 2^{-L \lambda}\left(\sum_{k=-\infty}^{L} 2^{k \alpha(0) q(0)}\left\|\sum_{l=k-1}^{k+1} \sum_{j=k-1}^{k+1} T\left(f_{1 l}, f_{2 j}\right) \chi_{k}\right\|_{L^{p(\cdot)}(w)}^{q(0)}\right)^{\frac{1}{q(0)}} \\
& E_{6}:=\sup _{L \leq 0, L \in \mathbb{Z}} 2^{-L \lambda}\left(\sum_{k=-\infty}^{L} 2^{k \alpha(0) q(0)}\left\|\sum_{l=k-1}^{k+1} \sum_{j=k+2}^{\infty} T\left(f_{1 l}, f_{2 j}\right) \chi_{k}\right\|_{L^{p(\cdot)}(w)}^{q(0)}\right)^{\frac{1}{q(0)}} \\
& E_{7}:=\sup _{L \leq 0, L \in \mathbb{Z}} 2^{-L \lambda}\left(\sum_{k=-\infty}^{L} 2^{k \alpha(0) q(0)}\left\|\sum_{l=k+2}^{\infty} \sum_{j=-\infty}^{k-2} T\left(f_{1 l}, f_{2 j}\right) \chi_{k}\right\|_{L^{p(\cdot)}(w)}^{q(0)}\right)^{\frac{1}{q(0)}} \\
& E_{8}:=\sup _{L \leq 0, L \in \mathbb{Z}} 2^{-L \lambda}\left(\sum_{k=-\infty}^{L} 2^{k \alpha(0) q(0)}\left\|\sum_{l=k+2}^{\infty} \sum_{j=k-1}^{k+1} T\left(f_{1 l}, f_{2 j}\right) \chi_{k}\right\|_{L^{p(\cdot)(w)}}^{q(0)}\right)^{\frac{1}{q(0)}} \\
& E_{9}:=\sup _{L \leq 0, L \in \mathbb{Z}} 2^{-L \lambda}\left(\sum_{k=-\infty}^{L} 2^{k \alpha(0) q(0)}\left\|\sum_{l=k+2}^{\infty} \sum_{j=k+2}^{\infty} T\left(f_{1 l}, f_{2 j}\right) \chi_{k}\right\|_{L^{p(\cdot)}(w)}^{q(0)}\right)^{\frac{1}{q(0)}} \\
& G_{1}:=\sup _{L>0, L \in \mathbb{Z}} 2^{-L \lambda}\left(\sum_{k=0}^{L} 2^{k \alpha_{\infty} q_{\infty}}\left\|\sum_{l=-\infty}^{k-2} \sum_{j=-\infty}^{k-2} T\left(f_{1 l}, f_{2 j}\right) \chi_{k}\right\|_{L^{p(\cdot)}(w)}^{q_{\infty}}\right)^{\frac{1}{q \infty}}, \\
& G_{2}:=\sup _{L>0, L \in \mathbb{Z}} 2^{-L \lambda}\left(\sum_{k=0}^{L} 2^{k \alpha_{\infty} q_{\infty}}\left\|\sum_{l=-\infty}^{k-2} \sum_{j=k-1}^{k+1} T\left(f_{1 l}, f_{2 j}\right) \chi_{k}\right\|_{L^{p(\cdot)}(w)}^{q_{\infty}}\right)^{\frac{1}{q \infty}}, \\
& G_{3}:=\sup _{L>0, L \in \mathbb{Z}} 2^{-L \lambda}\left(\sum_{k=0}^{L} 2^{k \alpha_{\infty} q_{\infty}}\left\|\sum_{l=-\infty}^{k-2} \sum_{j=k+2}^{\infty} T\left(f_{1 l}, f_{2 j}\right) \chi_{k}\right\|_{L^{p(\cdot)}(w)}^{q_{\infty}}\right)^{\frac{1}{q \infty}}, \\
& G_{4}:=\sup _{L>0, L \in \mathbb{Z}} 2^{-L \lambda}\left(\sum_{k=0}^{L} 2^{k \alpha_{\infty} q_{\infty}}\left\|\sum_{l=k-1}^{k+1} \sum_{j=-\infty}^{k-2} T\left(f_{1 l}, f_{2 j}\right) \chi_{k}\right\|_{L^{p(\cdot)}(w)}^{q_{\infty}}\right)^{\frac{1}{q \infty}}, \\
& G_{5}:=\sup _{L>0, L \in \mathbb{Z}} 2^{-L \lambda}\left(\sum_{k=0}^{L} 2^{k \alpha_{\infty} q_{\infty}}\left\|\sum_{l=k-1}^{k+1} \sum_{j=k-1}^{k+1} T\left(f_{1 l}, f_{2 j}\right) \chi_{k}\right\|_{L^{p(\cdot)}(w)}^{q_{\infty}}\right)^{\frac{1}{q \infty}}, \\
& G_{6}:=\sup _{L>0, L \in \mathbb{Z}} 2^{-L \lambda}\left(\sum_{k=0}^{L} 2^{k \alpha_{\infty} q \infty}\left\|\sum_{l=k-1}^{k+1} \sum_{j=k+2}^{\infty} T\left(f_{1 l}, f_{2 j}\right) \chi_{k}\right\|_{L^{p(\cdot)(w)}}^{q_{\infty}}\right)^{\frac{1}{q \infty}}, \\
& G_{7}:=\sup _{L>0, L \in \mathbb{Z}} 2^{-L \lambda}\left(\sum_{k=0}^{L} 2^{k \alpha_{\infty} q_{\infty}}\left\|\sum_{l=k+2}^{\infty} \sum_{j=-\infty}^{k-2} T\left(f_{1 l}, f_{2 j}\right) \chi_{k}\right\|_{L^{p(\cdot)}(w)}^{q_{\infty}}\right)^{\frac{1}{q \infty}},
\end{aligned}
$$




$$
\begin{aligned}
& G_{8}:=\sup _{L>0, L \in \mathbb{Z}} 2^{-L \lambda}\left(\sum_{k=0}^{L} 2^{k \alpha_{\infty} q_{\infty}}\left\|\sum_{l=k+2}^{\infty} \sum_{j=k-1}^{k+1} T\left(f_{1 l}, f_{2 j}\right) \chi_{k}\right\|_{L^{p(\cdot)(w)}}^{q_{\infty}}\right)^{\frac{1}{q \infty}}, \\
& G_{9}:=\sup _{L>0, L \in \mathbb{Z}} 2^{-L \lambda}\left(\sum_{k=0}^{L} 2^{k \alpha_{\infty} q_{\infty}}\left\|\sum_{l=k+2}^{\infty} \sum_{j=k+2}^{\infty} T\left(f_{1 l}, f_{2 j}\right) \chi_{k}\right\|_{L^{p(\cdot)(w)}}^{q_{\infty}}\right)^{\frac{1}{q \infty}} .
\end{aligned}
$$

We shall use the following estimates. If $l \leq k-1$, then, by Hölder's inequality and Lemmas 4 and 5 , we have

$$
\begin{aligned}
& \left\|2^{-k n} \int_{\mathbb{R}^{n}} f_{i l} d y_{i} \chi_{k}\right\|_{L^{p_{i}(\cdot)}\left(w_{i}\right)} \\
& \quad \leq C 2^{-k n}\left\|\chi_{B_{k}}\right\|_{L^{p_{i}(\cdot)}\left(w_{i}\right)}\left\|f_{i l} w_{i} \chi_{l}\right\|_{L^{p_{i}(\cdot)}}\left\|\chi_{l} w_{i}^{-1}\right\|_{L^{p_{i}^{\prime}(\cdot)}} \\
& \leq C 2^{-k n}\left|B_{k}\right|\left\|\chi_{B_{k}}\right\|_{L^{p_{i}^{\prime}(\cdot)}\left(w_{i}^{-1}\right)}\left\|\chi_{B_{l}}\right\|_{L_{i}^{p^{\prime} \cdot(\cdot)}\left(w_{i}^{-1}\right)}\left\|f_{i l} \chi_{l}\right\|_{L^{p_{i}(\cdot)}\left(w_{i}\right)} \\
& \leq C 2^{(l-k) n \delta_{2 i}}\left\|f_{i l} \chi_{l}\right\|_{L^{p_{i} \cdot(\cdot)}\left(w_{i}\right)} .
\end{aligned}
$$

If $l=k$, then

$$
\begin{aligned}
& \left\|2^{-k n} \int_{\mathbb{R}^{n}} f_{i l} d y_{i} \chi_{k}\right\|_{L^{p_{i}(\cdot)}\left(w_{i}\right)} \\
& \leq C 2^{-k n}\left\|\chi_{B_{k}}\right\|_{L^{p_{i}(\cdot)}\left(w_{i}\right)}\left\|f_{i l} w_{i} \chi_{l}\right\|_{L^{p_{i} \cdot(\cdot)}}\left\|\chi_{l} w_{i}^{-1}\right\|_{L^{p_{i}^{\prime}(\cdot)}} \\
& \leq C 2^{-k n}\left\|\chi_{B_{k}}\right\|_{L^{p^{(} \cdot(\cdot)}\left(w_{i}\right)}\left\|\chi_{B_{l}}\right\|_{L^{p_{i}^{\prime}(\cdot)}\left(w_{i}^{-1}\right)}\left\|f_{i l} \chi_{l}\right\|_{L^{p_{i}(\cdot)}\left(w_{i}\right)} \\
& \leq\left\|f_{i l} \chi_{l}\right\|_{L^{p_{i}(\cdot)}\left(w_{i}\right)} .
\end{aligned}
$$

If $l \geq k+1$, then

$$
\begin{aligned}
\| 2^{-k n} & \int_{\mathbb{R}^{n}} f_{i l} d y_{i} \chi_{k} \|_{L^{p_{i}(\cdot)}\left(w_{i}\right)} \\
\leq & C 2^{-k n}\left\|\chi_{B_{k}}\right\|_{L^{p_{i}(\cdot)}\left(w_{i}\right)}\left\|f_{i l} w_{i} \chi_{l}\right\|_{L^{p_{i}(\cdot)} \|}\left\|\chi_{l} w_{i}^{-1}\right\|_{L^{p_{i}^{\prime} \cdot(\cdot)}} \\
\leq & C 2^{-k n}\left\|\chi_{B_{k}}\right\|_{L^{p_{i}(\cdot)}\left(w_{i}\right)}\left\|\chi_{B_{l}}\right\|_{L^{p_{i} \cdot(\cdot)}\left(w_{i}\right)}\left\|\chi_{B_{l}}\right\|_{L^{p_{i}(\cdot)}\left(w_{i}\right)}^{-1} \\
& \times\left\|\chi_{B_{l}}\right\|_{L^{p_{i}^{\prime}(\cdot)}\left(w_{i}^{-1}\right)}\left\|f_{i l} \chi_{l}\right\|_{L^{p_{i}(\cdot)}\left(w_{i}\right)} \\
\leq & C 2^{(l-k) n\left(1-\delta_{1 i}\right)}\left\|f_{i l} \chi_{l}\right\|_{L^{p_{i}(\cdot)}\left(w_{i}\right)} .
\end{aligned}
$$

By the symmetry of $f_{1}$ and $f_{2}$, it is only necessary to estimate $E_{1}, E_{2}, E_{3}, E_{5}, E_{6}$, and $E_{9}$.

To estimate $E_{1}$, since $l, j \leq k-2$, we deduce that, for $i=1,2$,

$$
\left|x-y_{i}\right| \geq|x|-\left|y_{i}\right|>2^{k-1}-2^{\min \{l, j\}} \geq 2^{k-2}, \quad x \in D_{k}, y_{1} \in D_{l}, y_{2} \in D_{j} .
$$

Therefore, for $x \in D_{k}$, we have

$$
\left|K\left(x, y_{1}, y_{2}\right)\right| \leq C\left(\left|x-y_{1}\right|+\left|x-y_{2}\right|\right)^{-2 n} \leq C 2^{-2 k n} .
$$


Thus, $\forall x \in D_{k}$ and $l, j \leq k-2$, we have

$$
\begin{aligned}
\left|T\left(f_{1 l}, f_{2 j}\right)(x)\right| & \lesssim \int_{\mathbb{R}^{n}} \int_{\mathbb{R}^{n}} \frac{\left|f_{1 l}\left(y_{1}\right)\right|\left|f_{2 j}\left(y_{2}\right)\right|}{\left(\left|x-y_{1}\right|+\left|x-y_{2}\right|\right)^{2 n}} d y_{1} d y_{2} \\
& \lesssim 2^{-2 k n} \int_{\mathbb{R}^{n}} \int_{\mathbb{R}^{n}}\left|f_{1 l}\left(y_{1}\right)\right|\left|f_{2 j}\left(y_{2}\right)\right| d y_{1} d y_{2} .
\end{aligned}
$$

Therefore, by Hölder's inequality, we obtain

$$
\begin{aligned}
& \left\|\sum_{l=-\infty}^{k-2} \sum_{j=-\infty}^{k-2} T\left(f_{1 l}, f_{2 j}\right) \chi_{k}\right\|_{L^{p(\cdot)}(w)} \\
& \lesssim 2^{-2 k n}\left\|\sum_{l=-\infty}^{k-2} \sum_{j=-\infty}^{k-2} \int_{\mathbb{R}^{n}}\left|f_{1 l}\left(y_{1}\right)\right| d y_{1} \int_{\mathbb{R}^{n}}\left|f_{2 j}\left(y_{2}\right)\right| d y_{2} \chi_{k}\right\|_{L^{p(\cdot)}(w)} \\
& \lesssim\left\|\sum_{l=-\infty}^{k-2} 2^{-k n} \int_{\mathbb{R}^{n}}\left|f_{1 l}\left(y_{1}\right)\right| d y_{1} \chi_{k}\right\|_{L^{p_{1}(\cdot)}\left(w_{1}\right)} \\
& \quad \times\left\|\sum_{j=-\infty}^{k-2} 2^{-k n} \int_{\mathbb{R}^{n}}\left|f_{2 j}\left(y_{2}\right)\right| d y_{2} \chi_{k}\right\|_{L^{p_{2}(\cdot)}\left(w_{2}\right)} .
\end{aligned}
$$

Since $1 / q(0)=1 / q_{1}(0)+1 / q_{2}(0), \lambda=\lambda_{1}+\lambda_{2}$, by Hölder's inequality, we have

$$
\begin{aligned}
E_{1} \lesssim & \sup _{L \leq 0, L \in \mathbb{Z}} 2^{-L \lambda}\left(\sum_{k=-\infty}^{L} 2^{k \alpha(0) q(0)}\left\|\sum_{l=-\infty}^{k-2} 2^{-k n} \int_{\mathbb{R}^{n}}\left|f_{1 l}\left(y_{1}\right)\right| d y_{1} \chi_{k}\right\|_{L^{p_{1}(\cdot)}\left(w_{1}\right)}^{q(0)}\right. \\
& \times\left\|\sum_{j=-\infty}^{k-2} 2^{-k n} \int_{\mathbb{R}^{n}}\left|f_{2 j}\left(y_{2}\right)\right| d y_{2} \chi_{k}\right\|_{L^{p_{2}(\cdot)}\left(w_{2}\right)}^{q(0)} \\
\lesssim & \sup _{L \leq 0, L \in \mathbb{Z}} 2^{-L \lambda_{1}} \\
& \times\left(\sum_{k=-\infty}^{L(0)} 2^{k \alpha_{1}(0) q_{1}(0)}\left\|\sum_{l=-\infty}^{k-2} 2^{-k n} \int_{\mathbb{R}^{n}}\left|f_{1 l}\left(y_{1}\right)\right| d y_{1} \chi_{k}\right\|_{L^{p_{1}(\cdot)}\left(w_{1}\right)}^{q_{1}(0)}\right)^{\frac{1}{q_{1}(0)}} \\
& \times \sup _{L \leq 0, L \in \mathbb{Z}} 2^{-L \lambda_{2}} \\
& \times\left(\sum_{k=-\infty}^{L} 2^{k \alpha_{2}(0) q_{2}(0)}\left\|\sum_{j=-\infty}^{k-2} 2^{-k n} \int_{\mathbb{R}^{n}}\left|f_{2 j}\left(y_{2}\right)\right| d y_{2} \chi_{k}\right\|_{L^{p_{2}(\cdot)}\left(w_{2}\right)}^{q_{2}(0)}\right)^{\frac{1}{q_{2}(0)}} \\
:= & E_{1,1} \times E_{1,2},
\end{aligned}
$$

where

$$
\begin{aligned}
E_{1, i}:= & \sup _{L \leq 0, L \in \mathbb{Z}} 2^{-L \lambda_{i}} \\
& \times\left\{\sum_{k=-\infty}^{L} 2^{k \alpha_{i}(0) q_{i}(0)}\left\|\sum_{l=-\infty}^{k-2} 2^{-k n} \int_{\mathbb{R}^{n}}\left|f_{i l}\left(y_{i}\right)\right| d y_{i} \chi_{k}\right\|_{L^{p_{i}(\cdot)}\left(w_{i}\right)}^{q_{i}(0)}\right\}^{\frac{1}{q_{i}(0)}} .
\end{aligned}
$$


Since $n \delta_{2 i}-\alpha_{i}(0)>0$, by (5) and Lemma 7 we obtain

$$
\begin{aligned}
E_{1, i} \lesssim \sup _{L \leq 0, L \in \mathbb{Z}} 2^{-L \lambda_{i}}\left\{\sum_{k=-\infty}^{L} 2^{k \alpha_{i}(0) q_{i}(0)}\left(\sum_{l=-\infty}^{k-2} 2^{(l-k) n \delta_{2 i}}\left\|f_{i l}\right\|_{L^{p_{i}(\cdot)}\left(w_{i}\right)}\right)^{q_{i}(0)}\right\}^{\frac{1}{q_{i}(0)}} \\
=\sup _{L \leq 0, L \in \mathbb{Z}} 2^{-L \lambda_{i}} \\
\quad \times\left\{\sum_{k=-\infty}^{L}\left(\sum_{l=-\infty}^{k-2} 2^{l \alpha_{i}(0)}\left\|f_{i l}\right\|_{L^{p_{i}(\cdot)}\left(w_{i}\right)} 2^{(l-k)\left(n \delta_{2 i}-\alpha_{i}(0)\right)}\right)^{q_{i}(0)}\right\}^{\frac{1}{q_{i}(0)}} \\
\lesssim \sup _{L \leq 0, L \in \mathbb{Z}} 2^{-L \lambda_{i}}\left(\sum_{l=-\infty}^{L-2} 2^{l \alpha_{i}(0) q_{i}(0)}\left\|f_{i l}\right\|_{L^{p_{i}(\cdot)}\left(w_{i}\right)}^{q_{i}(0)}\right)^{\frac{1}{q_{i}(0)}} \\
\lesssim\left\|f_{i}\right\|_{M \dot{K}_{p_{i}(\cdot), \lambda_{i}}^{\alpha_{i}(\cdot), q_{i}(\cdot)}\left(w_{i}\right)}
\end{aligned}
$$

where we wrote $2^{-|k-l|\left(n \delta_{2 i}-\alpha_{i}(0)\right)} \lesssim 2^{-|k-l| \varepsilon_{i}}$ for some $\varepsilon_{i} \in\left(0, n \delta_{2 i}-\alpha_{i}(0)\right)$. Thus, we obtain

$$
E_{1} \lesssim\left\|f_{1}\right\|_{M \dot{K}_{p_{1}(\cdot), \lambda_{1}}^{\alpha_{1}(\cdot), q_{1}(\cdot)}\left(w_{1}\right)}\left\|f_{2}\right\|_{M \dot{K}_{p_{2}(\cdot), \lambda_{2}}^{\alpha_{2}(\cdot), q_{2}(\cdot)}\left(w_{2}\right)} \cdot
$$

To estimate $E_{2}$, since $l \leq k-2, k-1 \leq j \leq k+1$ for $i=1,2$, we have

$$
\left|x-y_{1}\right| \geq|x|-\left|y_{1}\right| \geq 2^{k-2}, \quad x \in D_{k}, y_{1} \in D_{l}
$$

Therefore, by Hölder's inequality, we obtain

$$
\begin{aligned}
& \left\|\sum_{l=-\infty}^{k-2} \sum_{j=k-1}^{k+1} T\left(f_{1 l}, f_{2 j}\right) \chi_{k}\right\|_{L^{p(\cdot)}(w)} \\
& \lesssim 2^{-2 k n}\left\|\sum_{l=-\infty}^{k-2} \sum_{j=k-1}^{k+1} \int_{\mathbb{R}^{n}}\left|f_{1 l}\left(y_{1}\right)\right| d y_{1} \int_{\mathbb{R}^{n}}\left|f_{2 j}\left(y_{2}\right)\right| d y_{2} \chi_{k}\right\|_{L^{p(\cdot)}(w)} \\
& \lesssim\left\|\sum_{l=-\infty}^{k-2} 2^{-k n} \int_{\mathbb{R}^{n}}\left|f_{1 l}\left(y_{1}\right)\right| d y_{1} \chi_{k}\right\|_{L^{p_{1}(\cdot)}\left(w_{1}\right)} \\
& \quad \times\left\|\sum_{j=k-1}^{k+1} 2^{-k n} \int_{\mathbb{R}^{n}}\left|f_{2 j}\left(y_{2}\right)\right| d y_{2} \chi_{k}\right\|_{L^{p_{2}(\cdot)}\left(w_{2}\right)} .
\end{aligned}
$$

Since $1 / q(0)=1 / q_{1}(0)+1 / q_{2}(0), \lambda=\lambda_{1}+\lambda_{2}$, by Hölder's inequality, we have

$$
\begin{aligned}
E_{2} \lesssim & \sup _{L \leq 0, L \in \mathbb{Z}} 2^{-L \lambda}\left(\sum_{k=-\infty}^{L} 2^{k \alpha(0) q(0)}\left\|\sum_{l=-\infty}^{k-2} 2^{-k n} \int_{\mathbb{R}^{n}}\left|f_{1 l}\left(y_{1}\right)\right| d y_{1} \chi_{k}\right\|_{L^{p_{1}(\cdot)}\left(w_{1}\right)}^{q(0)}\right. \\
& \left.\times\left\|\sum_{j=k-1}^{k+1} 2^{-k n} \int_{\mathbb{R}^{n}}\left|f_{2 j}\left(y_{2}\right)\right| d y_{2} \chi_{k}\right\|_{L^{p_{2}(\cdot)\left(w_{2}\right)}}^{q(0)}\right)^{\frac{1}{q(0)}} \\
& \lesssim \sup _{L \leq 0, L \in \mathbb{Z}} 2^{-L \lambda_{1}}
\end{aligned}
$$




$$
\begin{aligned}
& \times\left(\sum_{k=-\infty}^{L} 2^{k \alpha_{1}(0) q_{1}(0)}\left\|\sum_{l=-\infty}^{k-2} 2^{-k n} \int_{\mathbb{R}^{n}}\left|f_{1 l}\left(y_{1}\right)\right| d y_{1} \chi_{k}\right\|_{L^{p_{1}(\cdot)\left(w_{1}\right)}}^{q_{1}(0)}\right)^{\frac{1}{q_{1}(0)}} \\
& \times \sup _{L \leq 0, L \in \mathbb{Z}} 2^{-L \lambda_{2}} \\
& \times\left(\sum_{k=-\infty}^{L} 2^{k \alpha_{2}(0) q_{2}(0)}\left\|\sum_{j=k-1}^{k+1} 2^{-k n} \int_{\mathbb{R}^{n}}\left|f_{2 j}\left(y_{2}\right)\right| d y_{2} \chi_{k}\right\|_{L^{p_{2}(\cdot)\left(w_{2}\right)}}^{q_{2}(0)}\right)^{\frac{1}{q_{2}(0)}} \\
& :=E_{2,1} \times E_{2,2} .
\end{aligned}
$$

It is obvious that

$$
E_{2,1}=E_{1,1} \lesssim\left\|f_{1}\right\|_{M \dot{K}_{p_{1}}^{\alpha \cdot(\cdot), \lambda_{1}}}^{\alpha_{1}^{(\cdot), q_{1}(\cdot)}\left(w_{1}\right)}
$$

Now we estimate $E_{2,2}$. Taking (5), (6) and (7) together, we have

$$
\begin{aligned}
E_{2,2} & \lesssim \sup _{L \leq 0, L \in \mathbb{Z}} 2^{-L \lambda_{2}}\left(\sum_{k=-\infty}^{L} 2^{k \alpha_{2}(0) q_{2}(0)}\left\|\sum_{j=k-1}^{k+1} 2^{(j-k) n} f_{2 j}\right\|_{L^{p_{2}(\cdot)}\left(w_{2}\right)}^{q_{2}(0)}\right)^{\frac{1}{q_{2}(0)}} \\
& \lesssim \sup _{L \leq 0, L \in \mathbb{Z}} 2^{-L \lambda_{2}}\left(\sum_{k=-\infty}^{L} 2^{k \alpha_{2}(0) q_{2}(0)}\left\|f_{2 j}\right\|_{L^{p_{2}(\cdot)}\left(w_{2}\right)}^{q_{2}(0)}\right)^{\frac{1}{q_{2}(0)}} \\
& \lesssim\left\|f_{2}\right\|_{M \dot{K}_{p_{2}(\cdot), \lambda_{2}}^{\alpha_{2}(\cdot), q_{2}(\cdot)}\left(w_{2}\right)}
\end{aligned}
$$

where we used $2^{-n \delta_{22}}<1$ and $2^{(j-k) n\left(1-\delta_{12}\right)}<2^{(j-k) n}, j \in\{k-1, k, k+1\}$ for (5) and (7), respectively. Thus, we obtain

$$
E_{2} \lesssim\left\|f_{1}\right\|_{M \dot{K}_{p_{1}(\cdot), \lambda_{1}}^{\alpha_{1}(\cdot), q_{1}(\cdot)\left(w_{1}\right)}}\left\|f_{2}\right\|_{M \dot{K}_{p_{2}(\cdot), \lambda_{2}}^{\alpha_{2}(\cdot), q_{2}(\cdot)}\left(w_{2}\right)} \cdot
$$

To estimate $E_{3}$, since $l \leq k-2, j \geq k+2$, then we have

$$
\left|x-y_{1}\right| \geq|x|-\left|y_{1}\right| \geq 2^{k-2}, \quad\left|x-y_{2}\right| \geq\left|y_{2}\right|-|x|>2^{j-2}, \quad x \in D_{k}, y_{1} \in D_{l}, y_{2} \in D_{j} .
$$

Therefore, $\forall x \in D_{k}, l \leq k-2, j \geq k+2$, we get

$$
\begin{aligned}
\left|T\left(f_{1 l}, f_{2 j}\right)(x)\right| & \lesssim \int_{\mathbb{R}^{n}} \int_{\mathbb{R}^{n}} \frac{\left|f_{1 l}\left(y_{1}\right)\right|\left|f_{2 j}\left(y_{2}\right)\right|}{\left(\left|x-y_{1}\right|+\left|x-y_{2}\right|\right)^{2 n}} d y_{1} d y_{2} \\
& \lesssim 2^{-k n} 2^{-j n} \int_{\mathbb{R}^{n}} \int_{\mathbb{R}^{n}}\left|f_{1 l}\left(y_{1}\right)\right|\left|f_{2 j}\left(y_{2}\right)\right| d y_{1} d y_{2} .
\end{aligned}
$$

Thus, by Hölder's inequality, we have

$$
\begin{aligned}
& \left\|\sum_{l=-\infty}^{k-2} \sum_{j=k+2}^{\infty} T\left(f_{1 l}, f_{2 j}\right) \chi_{k}\right\|_{L^{p(\cdot)}(w)} \\
& \quad \lesssim 2^{-k n} 2^{-j n}\left\|\sum_{l=-\infty}^{k-2} \int_{\mathbb{R}^{n}}\left|f_{1 l}\left(y_{1}\right)\right| d y_{1} \sum_{j=k+2}^{\infty} \int_{\mathbb{R}^{n}}\left|f_{2 j}\left(y_{2}\right)\right| d y_{2} \chi_{k}\right\|_{L^{p(\cdot)}(w)}
\end{aligned}
$$




$$
\begin{aligned}
\lesssim & \left\|\sum_{l=-\infty}^{k-2} 2^{-k n} \int_{\mathbb{R}^{n}}\left|f_{1 l}\left(y_{1}\right)\right| d y_{1} \chi_{k}\right\|_{L^{p^{1}(\cdot)\left(w_{1}\right)}} \\
& \times\left\|\sum_{j=k+2}^{\infty} 2^{-j n} \int_{\mathbb{R}^{n}}\left|f_{2 j}\left(y_{2}\right)\right| d y_{2} \chi_{k}\right\|_{L^{p_{2}(\cdot)\left(w_{2}\right)}} .
\end{aligned}
$$

Since $1 / q(0)=1 / q_{1}(0)+1 / q_{2}(0), \lambda=\lambda_{1}+\lambda_{2}$, by Hölder's inequality, we have

$$
\begin{aligned}
E_{3} \lesssim & \sup _{L \leq 0, L \in \mathbb{Z}} 2^{-L \lambda}\left(\sum_{k=-\infty}^{L} 2^{k \alpha(0) q(0)}\left\|\sum_{l=-\infty}^{k-2} 2^{-k n} \int_{\mathbb{R}^{n}}\left|f_{1 l}\left(y_{1}\right)\right| d y_{1} \chi_{k}\right\|_{L^{p_{1}(\cdot)}\left(w_{1}\right)}^{q(0)}\right. \\
& \left.\times\left\|\sum_{j=k+2}^{\infty} 2^{-j n} \int_{\mathbb{R}^{n}}\left|f_{2 j}\left(y_{2}\right)\right| d y_{2} \chi_{k}\right\|_{L^{p_{2}(\cdot)\left(w_{2}\right)}}^{q(0)}\right)^{\frac{1}{q(0)}} \\
\lesssim & \sup _{L \leq 0, L \in \mathbb{Z}} 2^{-L \lambda_{1}} \\
& \times\left(\sum_{k=-\infty}^{L} 2^{k \alpha_{1}(0) q_{1}(0)}\left\|\sum_{l=-\infty}^{k-2} 2^{-k n} \int_{\mathbb{R}^{n}}\left|f_{1 l}\left(y_{1}\right)\right| d y_{1} \chi_{k}\right\|_{L^{p_{1}(\cdot)}\left(w_{1}\right)}^{q_{1}(0)}\right)^{\frac{1}{q_{1}(0)}} \\
& \times \sup _{L \leq 0, L \in \mathbb{Z}} 2^{-L \lambda_{2}} \\
& \times\left(\sum_{k=-\infty}^{L} 2^{k \alpha_{2}(0) q_{2}(0)}\left\|\sum_{j=k+2}^{\infty} 2^{-j n} \int_{\mathbb{R}^{n}}\left|f_{2 j}\left(y_{2}\right)\right| d y_{2} \chi_{k}\right\|_{L^{p_{2}(\cdot)}\left(w_{2}\right)}^{q_{2}(0)}\right)^{\frac{1}{q_{2}(0)}} \\
:= & E_{3,1} \times E_{3,2} .
\end{aligned}
$$

It is obvious that

$$
E_{3,1}=E_{1,1} \lesssim\left\|f_{1}\right\|_{M \dot{K}_{p_{1}(\cdot), \lambda_{1}}^{\alpha_{(}(\cdot), q_{1}(\cdot)}\left(w_{1}\right)} .
$$

Since $n \delta_{12}+\alpha_{2}(0)>0$, by (7) and Lemma 7 we obtain

$$
\begin{aligned}
E_{3,2} \lesssim & \sup _{L \leq 0, L \in \mathbb{Z}} 2^{-L \lambda_{2}}\left(\sum_{k=-\infty}^{L} 2^{k \alpha_{2}(0) q_{2}(0)}\right. \\
& \left.\times\left(\sum_{j=k+2}^{\infty} 2^{(k-j) n \delta_{12}}\left\|f_{2 j}\right\|_{L^{p_{2}(\cdot)}\left(w_{2}\right)}\right)^{q_{2}(0)}\right)^{\frac{1}{q_{2}(0)}} \\
\lesssim & \sup _{L \leq 0, L \in \mathbb{Z}} 2^{-L \lambda_{2}} \\
& \times\left(\sum_{k=-\infty}^{L}\left(\sum_{j=k+2}^{\infty} 2^{j \alpha_{2}(0)}\left\|f_{2 j}\right\|_{L^{p_{2}(\cdot)}\left(w_{2}\right)^{2}} 2^{(k-j)\left(n \delta_{12}+\alpha_{2}(0)\right)}\right)^{q_{2}(0)}\right)^{\frac{1}{q_{2}(0)}} \\
\lesssim & \sup _{L \leq 0, L \in \mathbb{Z}} 2^{-L \lambda_{2}}\left(\sum_{j=-\infty}^{L+2} 2^{j \alpha_{2}(0) q_{2}(0)}\left\|f_{2 j}\right\|_{L^{p_{2}(\cdot)}\left(w_{2}\right)}^{q_{2}(0)}\right)^{\frac{1}{q_{2}(0)}} \\
\lesssim & \left\|f_{2}\right\|_{M \dot{K}_{p_{2}(\cdot), \lambda_{2}}^{\alpha_{2}(\cdot), q_{2}(\cdot)}\left(w_{2}\right)^{\prime}}
\end{aligned}
$$


Wang and Xu Journal of Inequalities and Applications

(2019) 2019:251

Page 15 of 23

where we wrote $2^{-|k-j|\left(n \delta_{12}+\alpha_{2}(0)\right)} \lesssim 2^{-|k-j| \eta_{2}}$ for some $\eta_{2} \in\left(0, n \delta_{12}+\alpha_{2}(0)\right)$. Thus, we have

$$
E_{3} \lesssim\left\|f_{1}\right\|_{M \dot{K}_{p_{1}(\cdot), \lambda_{1}}^{\alpha_{1}(\cdot), q_{1}(\cdot)}\left(w_{1}\right)}\left\|f_{2}\right\|_{M \dot{K}_{p_{2}(\cdot), \lambda_{2}}^{\alpha_{2}(\cdot), q_{2}(\cdot)}\left(w_{2}\right)^{*}}
$$

To estimate $E_{5}$, using Hölder's inequality and Lemma 8 , we have

$$
\begin{aligned}
E_{5} \lesssim \sup _{L \leq 0, L \in \mathbb{Z}} 2^{-L \lambda}\left(\sum_{k=-\infty}^{L} 2^{k \alpha(0) q(0)}\left\|\sum_{l=k-1}^{k+1} \sum_{j=k-1}^{k+1} T\left(f_{1 l}, f_{2 j}\right) \chi_{k}\right\|_{L^{p(\cdot)}(w)}^{q(0)}\right)^{\frac{1}{q(0)}} \\
\lesssim \sup _{L \leq 0, L \in \mathbb{Z}} 2^{-L \lambda}\left(\sum_{k=-\infty}^{L} 2^{k \alpha(0) q(0)}\left(\left\|f_{1}\right\|_{L^{p_{1}(\cdot)}\left(w_{1}\right)}\left\|f_{2}\right\|_{L^{p_{2}(\cdot)}\left(w_{2}\right)}\right)^{q(0)}\right)^{\frac{1}{q(0)}} \\
\lesssim \sup _{L \leq 0, L \in \mathbb{Z}} 2^{-L \lambda_{1}}\left(\sum_{k=-\infty}^{L} 2^{k \alpha_{1}(0) q_{1}(0)}\left\|f_{1}\right\|_{L^{p_{1}(\cdot)}\left(w_{1}\right)}^{q_{1}(0)}\right)^{\frac{1}{q_{1}(0)}} \\
\quad \times 2^{-L \lambda_{2}}\left(\sum_{k=-\infty}^{L} 2^{k \alpha_{2}(0) q_{2}(0)}\left\|f_{2}\right\|_{L^{p_{2}(\cdot)}\left(w_{2}\right)}^{q_{2}(0)}\right)^{\frac{1}{q_{2}(0)}} \\
\lesssim\left\|f_{1}\right\|_{M \dot{K}_{p_{1}(\cdot), \lambda_{1}}^{\alpha_{1}(\cdot), q_{1}(\cdot)}\left(w_{1}\right)}\left\|f_{2}\right\|_{M \dot{K}_{p_{2}(\cdot), \lambda_{2}}^{\alpha_{2}(\cdot), q_{2}(\cdot)}\left(w_{2}\right)^{\cdot}}
\end{aligned}
$$

To estimate $E_{6}$, since $k-1 \leq l \leq k+1$ and $j \geq k+2$, we obtain

$$
\left|x-y_{1}\right|>2^{k-2}, \quad\left|x-y_{2}\right|>2^{j-2}, \quad x \in D_{k}, y_{1} \in D_{l}, y_{2} \in D_{j} .
$$

Thus, $\forall x \in D_{k}, k-1 \leq l \leq k+1$ and $j \geq k+2$, we obtain

$$
\begin{aligned}
\left|T\left(f_{1 l}, f_{2 j}\right)(x)\right| & \lesssim \int_{\mathbb{R}^{n}} \int_{\mathbb{R}^{n}} \frac{\left|f_{1 l}\left(y_{1}\right)\right|\left|f_{2 j}\left(y_{2}\right)\right|}{\left(\left|x-y_{1}\right|+\left|x-y_{2}\right|\right)^{2 n}} d y_{1} d y_{2} \\
& \lesssim 2^{-k n} 2^{-j n} \int_{\mathbb{R}^{n}} \int_{\mathbb{R}^{n}}\left|f_{1 l}\left(y_{1}\right)\right|\left|f_{2 j}\left(y_{2}\right)\right| d y_{1} d y_{2} .
\end{aligned}
$$

Therefore, by Hölder's inequality, we obtain

$$
\begin{aligned}
& \left\|\sum_{l=k-1}^{k+1} \sum_{j=k+2}^{\infty} T\left(f_{1 l}, f_{2 j}\right) \chi_{k}\right\|_{L^{p(\cdot)}(w)} \\
& \lesssim 2^{-k n} 2^{-j n}\left\|\sum_{l=k-1}^{k+1} \int_{\mathbb{R}^{n}}\left|f_{1 l}\left(y_{1}\right)\right| d y_{1} \sum_{j=-\infty}^{k-2} \int_{\mathbb{R}^{n}}\left|f_{2 j}\left(y_{2}\right)\right| d y_{2} \chi_{k}\right\|_{L^{p(\cdot)}(w)} \\
& \lesssim\left\|\sum_{l=k-1}^{k+1} 2^{-k n} \int_{\mathbb{R}^{n}}\left|f_{1 l}\left(y_{1}\right)\right| d y_{1} \chi_{k}\right\|_{L^{p_{1}(\cdot)}\left(w_{1}\right)} \\
& \quad \times\left\|\sum_{j=-\infty}^{k-2} 2^{-j n} \int_{\mathbb{R}^{n}}\left|f_{2 j}\left(y_{2}\right)\right| d y_{2} \chi_{k}\right\|_{L^{p_{2}(\cdot)}\left(w_{2}\right)} .
\end{aligned}
$$


Since $1 / q(0)=1 / q_{1}(0)+1 / q_{2}(0), \lambda=\lambda_{1}+\lambda_{2}$, by Hölder's inequality, we have

$$
\begin{aligned}
& E_{6} \lesssim \sup _{L \leq 0, L \in \mathbb{Z}} 2^{-L \lambda}\left(\sum_{k=-\infty}^{L} 2^{k \alpha(0) q(0)}\left\|\sum_{l=k-1}^{k+1} 2^{-k n} \int_{\mathbb{R}^{n}}\left|f_{1 l}\left(y_{1}\right)\right| d y_{1} \chi_{k}\right\|_{L^{p_{1}(\cdot)}\left(w_{1}\right)}^{q(0)}\right. \\
& \left.\times\left\|\sum_{j=k+2}^{\infty} 2^{-j n} \int_{\mathbb{R}^{n}}\left|f_{2 j}\left(y_{2}\right)\right| d y_{2} \chi_{k}\right\|_{L^{p_{2}(\cdot)}\left(w_{2}\right)}^{q(0)}\right)^{\frac{1}{q(0)}} \\
& \lesssim \sup _{L \leq 0, L \in \mathbb{Z}} 2^{-L \lambda_{1}} \\
& \times\left(\sum_{k=-\infty}^{L} 2^{k \alpha_{1}(0) q_{1}(0)}\left\|\sum_{l=k-1}^{k+1} 2^{-k n} \int_{\mathbb{R}^{n}}\left|f_{1 l}\left(y_{1}\right)\right| d y_{1} \chi_{k}\right\|_{L^{p_{1}(\cdot)}\left(w_{1}\right)}^{q_{1}(0)}\right)^{\frac{1}{q_{1}(0)}} \\
& \times \sup _{L \leq 0, L \in \mathbb{Z}} 2^{-L \lambda_{2}} \\
& \times\left(\sum_{k=-\infty}^{L} 2^{k \alpha_{2}(0) q_{2}(0)}\left\|\sum_{j=k+2}^{\infty} 2^{-j n} \int_{\mathbb{R}^{n}}\left|f_{2 j}\left(y_{2}\right)\right| d y_{2} \chi_{k}\right\|_{L^{p_{2}(\cdot)\left(w_{2}\right)}}^{q_{2}(0)}\right)^{\frac{1}{q_{2}(0)}} \\
& :=E_{6,1} \times E_{6,2} \text {. }
\end{aligned}
$$

By the symmetry of $f_{1}$ and $f_{2}$, we can know that the estimate $E_{6,1}$ is similar to the estimated $E_{2,2}$ and $E_{6,2}=E_{3,2}$.

To estimate $E_{9}$, since $l, j \geq k+2$, for $i=1,2$, we get

$$
\left|x-y_{i}\right|>2^{k-2}, \quad x \in D_{k}, y_{1} \in D_{l}, y_{2} \in D_{j} .
$$

Therefore, $\forall x \in D_{k}, l, j \geq k+2$, we have

$$
\begin{aligned}
\left|T\left(f_{1 l}, f_{2 j}\right)(x)\right| & \lesssim \int_{\mathbb{R}^{n}} \int_{\mathbb{R}^{n}} \frac{\left|f_{1 l}\left(y_{1}\right)\right|\left|f_{2 j}\left(y_{2}\right)\right|}{\left(\left|x-y_{1}\right|+\left|x-y_{2}\right|\right)^{2 n}} d y_{1} d y_{2} \\
& \lesssim 2^{-\ln } 2^{-j n} \int_{\mathbb{R}^{n}} \int_{\mathbb{R}^{n}}\left|f_{1 l}\left(y_{1}\right)\right|\left|f_{2 j}\left(y_{2}\right)\right| d y_{1} d y_{2} .
\end{aligned}
$$

Thus, by Hölder's inequality, we have

$$
\begin{aligned}
& \left\|\sum_{l=k+2}^{\infty} \sum_{j=k+2}^{\infty} T\left(f_{1 l}, f_{2 j}\right) \chi_{k}\right\|_{L^{p(\cdot)}(w)} \\
& \lesssim 2^{-\ln 2^{-j n} \|}\left\|\sum_{l=k+2}^{\infty} \int_{\mathbb{R}^{n}}\left|f_{1 l}\left(y_{1}\right)\right| d y_{1} \sum_{j=k+2}^{\infty} \int_{\mathbb{R}^{n}}\left|f_{2 j}\left(y_{2}\right)\right| d y_{2} \chi_{k}\right\|_{L^{p(\cdot)}(w)} \\
& \lesssim\left\|\sum_{l=k+2}^{\infty} 2^{-l n} \int_{\mathbb{R}^{n}}\left|f_{1 l}\left(y_{1}\right)\right| d y_{1} \chi_{k}\right\|_{L^{p_{1}(\cdot)}\left(w_{1}\right)} \\
& \quad \times\left\|\sum_{j=k+2}^{\infty} 2^{-j n} \int_{\mathbb{R}^{n}}\left|f_{2 j}\left(y_{2}\right)\right| d y_{2} \chi_{k}\right\|_{L^{p_{2}(\cdot)}\left(w_{2}\right)} .
\end{aligned}
$$


Since $1 / q(0)=1 / q_{1}(0)+1 / q_{2}(0), \lambda=\lambda_{1}+\lambda_{2}$, by Hölder's inequality, we have

$$
\begin{aligned}
& E_{9} \lesssim \sup _{L \leq 0, L \in \mathbb{Z}} 2^{-L \lambda}\left(\sum_{k=-\infty}^{L} 2^{k \alpha(0) q(0)}\left\|\sum_{l=k+2}^{\infty} 2^{-l n} \int_{\mathbb{R}^{n}}\left|f_{1 l}\left(y_{1}\right)\right| d y_{1} \chi_{k}\right\|_{L^{p_{1}(\cdot)}\left(w_{1}\right)}^{q(0)}\right. \\
& \left.\times\left\|\sum_{j=k+2}^{\infty} 2^{-j n} \int_{\mathbb{R}^{n}}\left|f_{2 j}\left(y_{2}\right)\right| d y_{2} \chi_{k}\right\|_{L^{p_{2}(\cdot)}\left(w_{2}\right)}^{q(0)}\right)^{\frac{1}{q(0)}} \\
& \lesssim \sup _{L \leq 0, L \in \mathbb{Z}} 2^{-L \lambda_{1}} \\
& \times\left(\sum_{k=-\infty}^{L} 2^{k \alpha_{1}(0) q_{1}(0)}\left\|\sum_{l=k+2}^{\infty} 2^{-l n} \int_{\mathbb{R}^{n}}\left|f_{1 l}\left(y_{1}\right)\right| d y_{1} \chi_{k}\right\|_{L^{p_{1}(\cdot)\left(w_{1}\right)}}^{q_{1}(0)}\right)^{\frac{1}{q_{1}(0)}} \\
& \times \sup _{L \leq 0, L \in \mathbb{Z}} 2^{-L \lambda_{2}} \\
& \times\left(\sum_{k=-\infty}^{L} 2^{k \alpha_{2}(0) q_{2}(0)}\left\|\sum_{j=k+2}^{\infty} 2^{-j n} \int_{\mathbb{R}^{n}}\left|f_{2 j}\left(y_{2}\right)\right| d y_{2} \chi_{k}\right\|_{L^{p_{2}(\cdot)}\left(w_{2}\right)}^{q_{2}(0)}\right)^{\frac{1}{q_{2}(0)}} \\
& :=E_{9,1} \times E_{9,2} \text {. }
\end{aligned}
$$

Obviously, the estimate $E_{9, i}$ is similar to the estimated $E_{3,2}$ for $i=1,2$.

Taking all estimates for $E_{i}$ together, $i=1,2, \ldots, 9$, we obtain

$$
E \lesssim\left\|f_{1}\right\|_{M \dot{K}_{p_{1}(\cdot), \lambda_{1}}^{\alpha_{1}(\cdot), q_{1}(\cdot)}\left(w_{1}\right)}\left\|f_{2}\right\|_{M \dot{K}_{p_{2}(\cdot), \lambda_{2}}^{\alpha_{2}(\cdot), q_{2}(\cdot)}\left(w_{2}\right)^{*}} .
$$

To go on, we need some further preparation.

If $l<0$, by Proposition 1 , we have

$$
\begin{aligned}
\left\|f_{i l} \chi_{l}\right\|_{L^{p_{i}(\cdot)}\left(w_{i}\right)} & =2^{-l \alpha_{i}(0)}\left(2^{l \alpha_{i}(0) q_{i}(0)}\left\|f_{i l} \chi_{l}\right\|_{L^{p_{i}(\cdot)}\left(w_{i}\right)}^{q_{i}(0)}\right)^{\frac{1}{q_{i}(0)}} \\
& \lesssim 2^{-l \alpha_{i}(0)}\left(\sum_{t=-\infty}^{l} 2^{t \alpha_{i}(0) q_{i}(0)}\left\|f_{i t} \chi_{t}\right\|_{L^{p_{i}(\cdot)}\left(w_{i}\right)}^{q_{i}(0)}\right)^{\frac{1}{q_{i}(0)}} \\
& \lesssim 2^{l\left(\lambda_{i}-\alpha_{i}(0)\right)}\left(2^{-l \lambda_{i}}\left(\sum_{t=-\infty}^{l}\left\|2^{t \alpha_{i}(0)} f_{i t} \chi_{t}\right\|_{L^{p_{i}(\cdot)}\left(w_{i}\right)}^{q_{i}(0)}\right)\right)^{\frac{1}{q_{i}(0)}} \\
& \lesssim 2^{l\left(\lambda_{i}-\alpha_{i}(0)\right)}\left\|f_{i}\right\|_{M \dot{K}_{p_{i}(\cdot), \lambda_{i}}^{\alpha_{i}(\cdot), q_{i}(\cdot)}\left(w_{i}\right)^{.}}
\end{aligned}
$$

If $l \geq 0$, we have

$$
\begin{aligned}
\left\|f_{i l} \chi_{l}\right\|_{L^{p_{i}(\cdot)}\left(w_{i}\right)} & =2^{-l \alpha_{i \infty}}\left(2^{l \alpha_{i \infty} q_{i \infty}}\left\|f_{i} \chi_{i l}\right\|_{L^{p(\cdot)}\left(w_{i}\right)}^{q_{i \infty}}\right)^{1 / q_{i \infty}} \\
& \lesssim 2^{-l \alpha_{i \infty}}\left(\sum_{t=-\infty}^{l} 2^{t \alpha_{i \infty} q_{i \infty}}\left\|f_{i t} \chi_{t}\right\|_{L^{p_{i}(\cdot)}\left(w_{i}\right)}^{q_{i \infty}}\right)^{1 / q_{i \infty}} \\
& \lesssim 2^{l\left(\lambda_{i}-\alpha_{i \infty}\right)}\left(2^{-l \lambda_{i}}\left(\sum_{t=-\infty}^{l}\left\|2^{t \alpha_{i \infty}} f_{i t} \chi_{t}\right\|_{L^{p_{i}(\cdot)}\left(w_{i}\right)}^{q_{i \infty}}\right)\right)^{1 / q_{i \infty}}
\end{aligned}
$$


Wang and Xu Journal of Inequalities and Applications

(2019) 2019:251

Page 18 of 23

$$
\lesssim 2^{l\left(\lambda_{i}-\alpha_{i \infty}\right)}\left\|f_{i}\right\|_{M \dot{K}_{p_{i}(\cdot), \lambda_{i}}^{\alpha_{i}(\cdot), q_{i}(\cdot)}\left(w_{i}\right)} .
$$

Finally, we estimate $G$. By the symmetry of $f_{1}$ and $f_{2}$, it is only necessary to estimate $G_{1}$, $G_{2}, G_{3}, G_{5}, G_{6}$, and $G_{9}$.

To estimate $G_{1}$, since $l, j \leq k-2,1 / q_{\infty}=1 / q_{1 \infty}+1 / q_{2 \infty}, \lambda=\lambda_{1}+\lambda_{2}$, by (8) and Hölder's inequality, we have

$$
\begin{aligned}
G_{1} \lesssim & \sup _{L>0, L \in \mathbb{Z}} 2^{-L \lambda}\left(\sum_{k=0}^{L} 2^{k \alpha_{\infty} q_{\infty}}\left\|\sum_{l=-\infty}^{k-2} 2^{-k n} \int_{\mathbb{R}^{n}}\left|f_{1 l}\left(y_{1}\right)\right| d y_{1} \chi_{k}\right\|_{L^{p_{1}(\cdot)\left(w_{1}\right)}}^{q_{\infty}}\right. \\
& \left.\times\left\|\sum_{j=-\infty}^{k-2} 2^{-k n} \int_{\mathbb{R}^{n}}\left|f_{2 j}\left(y_{2}\right)\right| d y_{2} \chi_{k}\right\|_{L^{p_{2}(\cdot)}\left(w_{2}\right)}^{q \infty}\right)^{\frac{1}{q \infty}} \\
\lesssim & \sup _{L>0, L \in \mathbb{Z}} 2^{-L \lambda_{1}} \\
& \times\left(\sum_{k=0}^{L} 2^{k \alpha_{1 \infty} q_{1 \infty}}\left\|\sum_{l=-\infty}^{k-2} 2^{-k n} \int_{\mathbb{R}^{n}}\left|f_{1 l}\left(y_{1}\right)\right| d y_{1} \chi_{k}\right\|_{L^{p_{1}(\cdot)\left(w_{1}\right)}}^{q_{1 \infty}}\right)^{\frac{1}{q_{1 \infty}}} \\
& \times \sup _{L>0, L \in \mathbb{Z}} 2^{-L \lambda_{2}} \\
& \times\left(\sum_{k=0}^{L} 2^{k \alpha_{2 \infty} q_{2 \infty}}\left\|\sum_{j=-\infty}^{k-2} 2^{-k n} \int_{\mathbb{R}^{n}}\left|f_{2 j}\left(y_{2}\right)\right| d y_{2} \chi_{k}\right\|_{L^{p_{2}(\cdot)\left(w_{2}\right)}}^{q_{2 \infty}}\right)^{\frac{1}{q_{2 \infty}}} \\
:= & G_{1,1} \times G_{1,2},
\end{aligned}
$$

where

$$
G_{1, i}:=\sup _{L>0, L \in \mathbb{Z}} 2^{-L \lambda_{i}}\left(\sum_{k=0}^{L} 2^{k \alpha_{i \infty} q_{i \infty}}\left\|\sum_{l=-\infty}^{k-2} 2^{-k n} \int_{\mathbb{R}^{n}}\left|f_{i l}\left(y_{i}\right)\right| d y_{i} \chi_{k}\right\|_{L^{p_{i}(\cdot)}\left(w_{i}\right)}^{q_{i \infty}}\right)^{\frac{1}{q_{i \infty}}} .
$$

Since $\lambda_{i}+n \delta_{2 i}>\alpha_{i \infty} \geq \alpha_{i}(0)$, by (5), (13), (14) and Lemma 7 we obtain

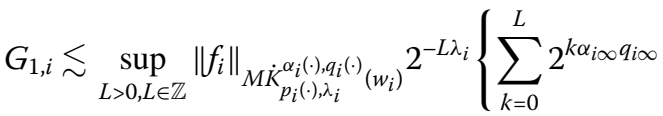

$$
\begin{aligned}
& \left.\times\left(\sum_{l=-\infty}^{-1} 2^{(l-k) n \delta_{2 i}} 2^{l\left(\lambda_{i}-\alpha_{i}(0)\right)}+\sum_{l=0}^{k} 2^{(l-k) n \delta_{12}} 2^{i\left(\lambda_{1}-\alpha_{1 \infty}\right)}\right)^{q_{i \infty}}\right\}^{\frac{1}{q_{i \infty}}} \\
& \lesssim \sup _{L>0, L \in \mathbb{Z}}\left\|f_{i}\right\|_{M K_{p_{i}(\cdot), \lambda_{i}}^{\alpha_{i}(\cdot), q_{i}(\cdot)}\left(w_{i}\right)} 2^{-L \lambda_{i}}\left\{\sum_{k=0}^{L} 2^{k \alpha_{i \infty} q_{i \infty}}\right. \\
& \times\left(\sum_{l=-\infty}^{-1} 2^{(l-k)\left(n \delta_{2 i}+\lambda_{i}-\alpha_{i \infty}\right)} 2^{l\left(\alpha_{i \infty}-\alpha_{i}(0)\right)} 2^{k\left(\lambda_{i}-\alpha_{i \infty}\right)}\right. \\
& \left.\left.+\sum_{l=0}^{k} 2^{(l-k)\left(n \delta_{2 i}+\lambda_{i}-\alpha_{i \infty}\right)} 2^{k\left(\lambda_{i}-\alpha_{i \infty}\right)}\right)^{q_{i \infty}}\right\}^{\frac{1}{q_{i \infty}}} \\
& \lesssim \sup _{L>0, L \in \mathbb{Z}}\left\|f_{i}\right\|_{M \dot{K}_{p_{i}}^{\alpha_{i}(\cdot), \lambda_{i}}} \cos _{i}(\cdot)\left(w_{i}\right)
\end{aligned}
$$




$$
\begin{aligned}
& \times 2^{-L \lambda_{i}}\left\{\sum_{k=0}^{L} 2^{k \lambda_{i} q_{i \infty}}\left(\sum_{l=-\infty}^{k} 2^{(l-k)\left(n \delta_{2 i}-\alpha_{i \infty}+\lambda_{i}\right)}\right)^{q_{i \infty}}\right\}^{\frac{1}{q_{i \infty}}} \\
\lesssim & \sup _{L>0, L \in \mathbb{Z}}\left\|f_{i}\right\|_{M \dot{K}_{p_{i}(\cdot), \lambda_{i}}^{\alpha_{i}(\cdot), q_{i}(\cdot)}\left(w_{i}\right)} 2^{-L \lambda_{i}}\left(\sum_{k=0}^{L} 2^{k \lambda_{i} q_{i \infty}}\right)^{\frac{1}{q_{i \infty}}} \\
\lesssim & \left\|f_{i}\right\|_{M \dot{K}_{p_{i}}^{\left.\alpha_{i}(\cdot),\right)_{i}, q_{i}(\cdot)}\left(w_{i}\right)^{*}} \cdot
\end{aligned}
$$

Thus, we get

$$
G_{1} \lesssim\left\|f_{1}\right\|_{M \dot{K}_{p_{1}}^{\alpha_{1}(\cdot),,_{1}}}{ }_{(\cdot), q_{1}(\cdot)}\left\|f_{\left.w_{1}\right)}\right\|_{M \dot{K}_{p_{2}(\cdot), \lambda_{2}}^{\alpha_{2}(\cdot), q_{2}(\cdot)}\left(w_{2}\right)} .
$$

To estimate $G_{2}$, since $l \leq k-2, k-1 \leq j \leq k+1,1 / q_{\infty}=1 / q_{1 \infty}+1 / q_{2 \infty}, \lambda=\lambda_{1}+\lambda_{2}$, by (9) and Hölder's inequality, we have

$$
\begin{aligned}
G_{2} \lesssim & \sup _{L>0, L \in \mathbb{Z}} 2^{-L \lambda}\left(\sum_{k=0}^{L} 2^{k \alpha_{\infty} q \infty}\left\|\sum_{l=-\infty}^{k-2} 2^{-k n} \int_{\mathbb{R}^{n}}\left|f_{1 l}\left(y_{1}\right)\right| d y_{1} \chi_{k}\right\|_{L^{p_{1}(\cdot)}\left(w_{1}\right)}^{q_{\infty}}\right. \\
& \left.\times\left\|\sum_{j=k-1}^{k+1} 2^{-k n} \int_{\mathbb{R}^{n}}\left|f_{2 j}\left(y_{2}\right)\right| d y_{2} \chi_{k}\right\|_{L^{p_{2}(\cdot)\left(w_{2}\right)}}^{q_{\infty}}\right)^{\frac{1}{q \infty}} \\
\lesssim & \sup _{L>0, L \in \mathbb{Z}} 2^{-L \lambda_{1}} \\
& \times\left(\sum_{k=0}^{L} 2^{k \alpha_{1 \infty} q_{1 \infty}}\left\|\sum_{l=-\infty}^{k-2} 2^{-k n} \int_{\mathbb{R}^{n}}\left|f_{1 l}\left(y_{1}\right)\right| d y_{1} \chi_{k}\right\|_{L^{p_{1}(\cdot)}\left(w_{1}\right)}^{q_{1 \infty}}\right)^{\frac{1}{q_{1 \infty}}} \\
& \times \sup _{L>0, L \in \mathbb{Z}} 2^{-L \lambda_{2}} \\
& \times\left(\sum_{k=0}^{L} 2^{k \alpha_{2 \infty} q_{2 \infty}}\left\|\sum_{j=k-1}^{k+1} 2^{-k n} \int_{\mathbb{R}^{n}}\left|f_{2 j}\left(y_{2}\right)\right| d y_{2} \chi_{k}\right\|_{L^{p_{2}(\cdot)\left(w_{2}\right)}}^{q_{2 \infty}}\right)^{\frac{1}{q_{2 \infty}}} \\
:= & G_{2,1} \times G_{2,2} .
\end{aligned}
$$

It is obvious that

$$
G_{2,1}=G_{1,1} \lesssim\left\|f_{1}\right\|_{M \dot{K}_{p_{1}(\cdot), \lambda_{1}}^{\alpha_{1}(\cdot), q_{1}(\cdot)}\left(w_{1}\right)} \cdot
$$

Now we estimate $G_{2,2}$. Combining (5), (6) and (7), we have

$$
\begin{aligned}
G_{2,2} & \lesssim \sup _{L>0, L \in \mathbb{Z}} 2^{-L \lambda_{2}}\left(\sum_{k=0}^{L} 2^{k \alpha_{2 \infty} q_{2 \infty}}\left\|\sum_{j=k-1}^{k+1} 2^{(j-k) n}\left|f_{2 j}\right|\right\|_{L^{p_{2}(\cdot)\left(w_{2}\right)}}^{q_{2 \infty}}\right)^{\frac{1}{q_{2 \infty}}} \\
& \lesssim \sup _{L>0, L \in \mathbb{Z}} 2^{-L \lambda_{2}}\left(\sum_{k=0}^{L} 2^{k \alpha_{2 \infty} q_{2 \infty}}\left\|\left|f_{2 j}\right|\right\|_{L^{p_{2}(\cdot)}\left(w_{2}\right)}^{q_{2 \infty}}\right)^{\frac{1}{q_{2 \infty}}} \\
& \lesssim\left\|f_{2}\right\|_{M \dot{K}_{p_{2}(\cdot), \lambda_{2}}^{\alpha_{2}(\cdot), q_{2}(\cdot)}\left(w_{2}\right)}
\end{aligned}
$$


where we used $2^{-n \delta_{22}}<1$ and $2^{(j-k) n\left(1-\delta_{12}\right)}<2^{(j-k) n}$ for (5) and (7), respectively. Thus, we obtain

$$
G_{2} \lesssim\left\|f_{1}\right\|_{M \dot{K}_{p_{1}}^{\alpha_{1}(\cdot), \lambda_{1}}\left(\cdot, q_{1}(\cdot)\right.}\left\|f_{\left.w_{1}\right)}\right\|_{M \dot{K}_{p_{2}(\cdot), \lambda_{2}}^{\alpha_{2}(\cdot), q_{2}(\cdot)}\left(w_{2}\right)} \cdot
$$

To estimate $G_{3}$, since $l \leq k-2, j \geq k+2,1 / q_{\infty}=1 / q_{1 \infty}+1 / q_{2 \infty}, \lambda=\lambda_{1}+\lambda_{2}$, by (10) and Hölder's inequality, we have

$$
\begin{aligned}
G_{3} \lesssim & \sup _{L>0, L \in \mathbb{Z}} 2^{-L \lambda}\left(\sum_{k=0}^{L} 2^{k \alpha_{\infty} q_{\infty}}\left\|\sum_{l=-\infty}^{k-2} 2^{-k n} \int_{\mathbb{R}^{n}}\left|f_{1 l}\left(y_{1}\right)\right| d y_{1} \chi_{k}\right\|_{L^{p_{1}(\cdot)\left(w_{1}\right)}}^{q_{\infty}}\right. \\
& \left.\times\left\|\sum_{j=k+2}^{\infty} 2^{-j n} \int_{\mathbb{R}^{n}}\left|f_{2 j}\left(y_{2}\right)\right| d y_{2} \chi_{k}\right\|_{L^{p_{2}(\cdot)\left(w_{2}\right)}}^{q_{\infty}}\right)^{\frac{1}{q \infty}} \\
\lesssim & \sup _{L>0, L \in \mathbb{Z}} 2^{-L \lambda_{1}} \\
& \times\left(\sum_{k=0}^{L} 2^{k \alpha_{1 \infty} q_{1 \infty}}\left\|\sum_{l=-\infty}^{k-2} 2^{-k n} \int_{\mathbb{R}^{n}}\left|f_{1 l}\left(y_{1}\right)\right| d y_{1} \chi_{k}\right\|_{L^{p_{1}(\cdot)}\left(w_{1}\right)}^{q_{1 \infty}}\right)^{\frac{1}{q_{1 \infty}}} \\
& \times \sup _{L>0, L \in \mathbb{Z}} 2^{-L \lambda_{2}} \\
& \times\left(\sum_{k=0}^{L} 2^{k \alpha_{2 \infty} q_{2 \infty}}\left\|\sum_{j=k+2}^{\infty} 2^{-j n} \int_{\mathbb{R}^{n}}\left|f_{2 j}\left(y_{2}\right)\right| d y_{2} \chi_{k}\right\|_{L^{p_{2}(\cdot)\left(w_{2}\right)}}^{q_{2 \infty}}\right)^{\frac{1}{q_{2 \infty}}} \\
:= & G_{3,1} \times G_{3,2} .
\end{aligned}
$$

It is obvious that

$$
G_{3,1}=G_{1,1} \lesssim\left\|f_{1}\right\|_{M \dot{K}_{p_{1}(\cdot), \lambda_{1}}^{\alpha_{1}(\cdot), q_{1}(\cdot)}\left(w_{1}\right)} \cdot
$$

Since $n \delta_{12}+\alpha_{2 \infty}>0$, by (7) and Lemma 7 we obtain

$$
\begin{aligned}
G_{3,2} & \lesssim \sup _{L>0, L \in \mathbb{Z}} 2^{-L \lambda_{2}}\left(\sum_{k=0}^{L} 2^{k \alpha_{2 \infty} q_{2 \infty}}\left(\sum_{j=k+2}^{\infty} 2^{(k-j) n \delta_{12}}\left\|f_{2 j}\right\|_{L^{p_{2}(\cdot)}\left(w_{2}\right)}\right)^{q_{2 \infty}}\right)^{\frac{1}{q_{2 \infty}}} \\
& \lesssim \sup _{L>0, L \in \mathbb{Z}} 2^{-L \lambda_{2}}\left(\sum_{k=0}^{L}\left(\sum_{j=k+2}^{\infty} 2^{j \alpha_{2 \infty}}\left\|f_{2 j}\right\|_{L^{p_{2}(\cdot)}\left(w_{2}\right)} 2^{(k-j)\left(n \delta_{12}+\alpha_{2 \infty}\right)}\right)^{q_{2 \infty}}\right)^{\frac{1}{q_{2 \infty}}} \\
& \lesssim \sup _{L>0, L \in \mathbb{Z}} 2^{-L \lambda_{2}}\left(\sum_{j=2}^{L+2} 2^{j \alpha_{2 \infty} q_{2 \infty}}\left\|f_{2 j}\right\|_{L^{p_{2}(\cdot)}\left(w_{2}\right)}^{q_{2 \infty}}\right)^{\frac{1}{q_{2 \infty}}} \\
& \lesssim\left\|f_{2}\right\|_{M \dot{K}_{p_{2}(\cdot), \lambda_{2}}^{\alpha_{2}(\cdot), q_{2}(\cdot)}\left(w_{2}\right)^{\prime}}
\end{aligned}
$$

where we wrote $2^{-|k-j| \mid\left(n \delta_{12}+\alpha_{2} \infty\right)} \lesssim 2^{-|k-j| \vartheta_{2}}$ for some $\vartheta_{2} \in\left(0, n \delta_{12}+\alpha_{2 \infty}\right)$. Thus, we get

$$
G_{3} \lesssim\left\|f_{1}\right\|_{M \dot{K}_{p_{1}(\cdot), \lambda_{1}}^{\alpha_{1}(\cdot), q_{1}(\cdot)}\left(w_{1}\right)}\left\|f_{2}\right\|_{M \dot{K}_{p_{2}(\cdot), \lambda_{2}}^{\alpha_{2}(\cdot), q_{2}(\cdot)}\left(w_{2}\right)} .
$$


To estimate $G_{5}$, using Hölder's inequality and Lemma 8

$$
\begin{aligned}
& G_{5} \lesssim \sup _{L>0, L \in \mathbb{Z}} 2^{-L \lambda}\left(\sum_{k=0}^{L} 2^{k \alpha_{\infty} q_{\infty}}\left\|\sum_{l=k-1}^{k+1} \sum_{j=k-1}^{k+1} T\left(f_{1 l}, f_{2 j}\right) \chi_{k}\right\|_{L^{p(\cdot)}(w)}^{q_{\infty}}\right)^{\frac{1}{q \infty}} \\
& \lesssim \sup _{L>0, L \in \mathbb{Z}} 2^{-L \lambda}\left(\sum_{k=0}^{L} 2^{k \alpha_{\infty} q_{\infty}}\left(\left\|f_{1}\right\|_{L^{p_{1}(\cdot)}\left(w_{1}\right)}\left\|f_{2}\right\|_{L^{p_{2}(\cdot)}\left(w_{2}\right)}\right)^{q_{\infty}}\right)^{\frac{1}{q \infty}} \\
& \lesssim \sup _{L>0, L \in \mathbb{Z}} 2^{-L \lambda_{1}}\left(\sum_{k=0}^{L} 2^{k \alpha_{1 \infty} q_{1 \infty}}\left\|f_{1}\right\|_{L^{p_{1}(\cdot)}\left(w_{1}\right)}^{q_{1 \infty}}\right)^{\frac{1}{q_{1 \infty}}} \\
& \times 2^{-L \lambda_{2}}\left(\sum_{k=0}^{L} 2^{k \alpha_{2 \infty} q_{2 \infty}}\left\|f_{2}\right\|_{L^{p_{2}(\cdot)}\left(w_{2}\right)}^{q_{2 \infty}}\right)^{\frac{1}{q_{2 \infty}}} \\
& \lesssim\left\|f_{1}\right\|_{M \dot{K}_{p_{1}(\cdot), \lambda_{1}}^{\alpha_{1}(\cdot), q_{1}(\cdot)}\left(w_{1}\right)}\left\|f_{2}\right\|_{M \dot{K}_{p_{2}(\cdot), \lambda_{2}}^{\alpha_{2}(\cdot), q_{2}(\cdot)}{ }_{\left(w_{2}\right)}} \text {. }
\end{aligned}
$$

To estimate $G_{6}$, since $k-1 \leq l \leq k+1$ and $j \geq k+2,1 / q_{\infty}=1 / q_{1 \infty}+1 / q_{2 \infty}, \lambda=\lambda_{1}+\lambda_{2}$, by (11) and Hölder's inequality, we have

$$
\begin{aligned}
G_{6} \lesssim & \sup _{L>0, L \in \mathbb{Z}} 2^{-L \lambda}\left(\sum_{k=0}^{L} 2^{k \alpha_{\infty} q_{\infty}}\left\|\sum_{l=k-1}^{k+1} 2^{-k n} \int_{\mathbb{R}^{n}}\left|f_{1 l}\left(y_{1}\right)\right| d y_{1} \chi_{k}\right\|_{L^{p_{1}(\cdot)}\left(w_{1}\right)}^{q_{\infty}}\right. \\
& \left.\times\left\|\sum_{j=k+2}^{\infty} 2^{-j n} \int_{\mathbb{R}^{n}}\left|f_{2 j}\left(y_{2}\right)\right| d y_{2} \chi_{k}\right\|_{L^{p_{2}(\cdot)\left(w_{2}\right)}}^{q_{\infty}}\right)^{\frac{1}{q_{\infty}}} \\
\lesssim & \sup _{L>0, L \in \mathbb{Z}} 2^{-L \lambda_{1}} \\
& \times\left(\sum_{k=0}^{L} 2^{k \alpha_{1 \infty} q_{1 \infty}}\left\|\sum_{l=k-1}^{k+1} 2^{-k n} \int_{\mathbb{R}^{n}}\left|f_{1 l}\left(y_{1}\right)\right| d y_{1} \chi_{k}\right\|_{L^{p_{1}(\cdot)\left(w_{1}\right)}}^{q_{1 \infty}}\right)^{\frac{1}{q_{1 \infty}}} \\
& \times \sup _{L>0, L \in \mathbb{Z}} 2^{-L \lambda_{2}} \\
& \times\left(\sum_{k=0}^{L} 2^{k \alpha_{2 \infty} q_{2 \infty}}\left\|\sum_{j=k+2}^{\infty} 2^{-j n} \int_{\mathbb{R}^{n}}\left|f_{2 j}\left(y_{2}\right)\right| d y_{2} \chi_{k}\right\|_{L^{p_{2}(\cdot)\left(w_{2}\right)}}^{q_{2 \infty}}\right)^{\frac{1}{q_{2 \infty}}} \\
:= & G_{6,1} \times G_{6,2 .}
\end{aligned}
$$

By the symmetry of $f_{1}$ and $f_{2}$, we can know that the estimate $G_{6,1}$ is similar to the estimated $G_{2,2}$ and $G_{6,2}=G_{3,2}$.

To estimate $G_{9}$, since $l, j \geq k+2,1 / q_{\infty}=1 / q_{1 \infty}+1 / q_{2 \infty}, \lambda=\lambda_{1}+\lambda_{2}$, by (12) and Hölder's inequality, we have

$$
\begin{aligned}
G_{9} \lesssim & \sup _{L>0, L \in \mathbb{Z}} 2^{-L \lambda}\left(\sum_{k=0}^{L} 2^{k \alpha_{\infty} q_{\infty}}\left\|\sum_{l=k+2}^{\infty} 2^{-l n} \int_{\mathbb{R}^{n}}\left|f_{1 l}\left(y_{1}\right)\right| d y_{1} \chi_{k}\right\|_{L^{p_{1}(\cdot)\left(w_{1}\right)}}^{q_{\infty}}\right. \\
& \left.\times\left\|\sum_{j=k+2}^{\infty} 2^{-j n} \int_{\mathbb{R}^{n}}\left|f_{2 j}\left(y_{2}\right)\right| d y_{2} \chi_{k}\right\|_{L^{p_{2}(\cdot)\left(w_{2}\right)}}^{q_{\infty}}\right)^{\frac{1}{q_{\infty}}}
\end{aligned}
$$




$$
\begin{aligned}
& \lesssim \sup _{L>0, L \in \mathbb{Z}} 2^{-L \lambda_{1}} \\
& \quad \times\left(\sum_{k=0}^{L} 2^{k \alpha_{1} \infty q_{1} \infty}\left\|\sum_{l=k+2}^{\infty} 2^{-l n} \int_{\mathbb{R}^{n}}\left|f_{1 l}\left(y_{1}\right)\right| d y_{1} \chi_{k}\right\|_{L^{p_{1}(\cdot)\left(w_{1}\right)}}^{q_{1 \infty}}\right)^{\frac{1}{q_{1 \infty}}} \\
& \quad \times \sup _{L>0, L \in \mathbb{Z}} 2^{-L \lambda_{2}} \\
& \quad \times\left(\sum_{k=0}^{L} 2^{k \alpha_{2 \infty} q_{2 \infty}}\left\|\sum_{j=k+2}^{\infty} 2^{-j n} \int_{\mathbb{R}^{n}}\left|f_{2 j}\left(y_{2}\right)\right| d y_{2} \chi_{k}\right\|_{L^{p_{2}(\cdot)}\left(w_{2}\right)}^{q_{2 \infty}}\right)^{\frac{1}{q_{2 \infty}}} \\
& :=G_{9,1} \times G_{9,2} .
\end{aligned}
$$

Obviously, the estimate $G_{9, i}$ is similar to the estimated $G_{3,2}$ for $i=1,2$.

Taking all estimates for $G_{i}$ together, $i=1,2, \ldots, 9$, we obtain

$$
G \lesssim\left\|f_{1}\right\|_{M \dot{K}_{p_{1}(\cdot), \lambda_{1}}^{\alpha_{1}(\cdot), q_{1}(\cdot)}\left(w_{1}\right)}\left\|f_{2}\right\|_{M \dot{K}_{p_{2}(\cdot), \lambda_{2}}^{\alpha_{2}(\cdot), q_{2}(\cdot)}\left(w_{2}\right)^{*}} .
$$

This completes the proof.

\section{Acknowledgements}

The authors would like to thank the referees for their careful reading and suggestions.

\section{Funding}

The work is partially supported by Hainan Province Natural Science Foundation of China (2018CXTD338) and the National Natural Science Foundation of China (Grant No. 11761026 and 11761027).

\section{Availability of data and materials}

Not applicable.

\section{Competing interests}

The authors declare that they have no competing interests.

\section{Authors' contributions}

All authors contributed equally and significantly in writing this paper. All authors read and approved the final manuscript.

\section{Author details}

${ }^{1}$ School of Mathematics and Statistics, Hainan Normal University, Haikou, China. ${ }^{2}$ School of Mathematics and Computing Science, Guilin University of Electronic Technology, Guilin, China.

\section{Publisher's Note}

Springer Nature remains neutral with regard to jurisdictional claims in published maps and institutional affiliations.

Received: 4 April 2019 Accepted: 6 September 2019 Published online: 23 September 2019

\section{References}

1. Cruz-Uribe, D., Guzman, O.M.: Weighted norm inequalities for the bilinear maximal operator on variable Lebesgue spaces (2018) https://arxiv.org/abs/1811.00618

2. Izuki, M.: Herz and amalgam spaces with variable exponent, the Haar wavelets and greediness of the wavelet system. East J. Approx. 15(1), 87-109 (2009)

3. Izuki, M.: Boundedness of sublinear operators on Herz spaces with variable exponent and application to wavelet characterization. Anal. Math. 36(1), 33-50 (2010)

4. Izuki, M.: Commutators of fractional integrals on Lebesgue and Herz spaces with variable exponent. Rend. Circ. Mat. Palermo 59(3), 461-472 (2010)

5. Izuki, M.: Fractional integrals on Herz-Morrey spaces with variable exponent. Hiroshima Math. J. 40(3), 343-355 (2010)

6. Izuki, M., Noi, T.: Hardy spaces associated to critical Herz spaces with variable exponent. Mediterr. J. Math. 13(5), $2981-3013(2016)$

7. Izuki, M., Noi, T.: Boundedness of fractional integrals on weighted Herz spaces with variable exponent. J. Inequal. Appl. 2016(199), 15 (2016) 
8. Izuki, M.: Boundedness of commutators on Herz spaces with variable exponent. Rend. Circ. Mat. Palermo 59(2), 199-213 (2010)

9. Izuki, M., Noi, T.: An intrinsic square function on weighted Herz spaces with variable exponent. J. Math. Inequal. 11(3), 799-816 (2017)

10. Wang, L.W., Shu, L.S.: Boundedness of some sublinear operators on weighted variable Herz-Morrey spaces. J. Math. Inequal. 12(1), 31-42 (2018)

11. Wang, P., Liu, Z:: Weighted norm inequalities for multilinear Calderón-Zygmund operators in generalized Morrey spaces. J. Inequal. Appl. 2017, Article ID 48 (2017)

12. Coifman, R.R., Meyer, Y.: On commutators of singular integrals and bilinear singular integrals. Trans. Am. Math. Soc. 212, 315-331 (1975)

13. Bui, T.A., Duong, X.T.: Weighted norm inequalities for multilinear operators and applications to multilinear Fourier multipliers. Bull. Sci. Math. 137(1), 63-75 (2013)

14. Grafakos, L., Kalton, N.: Multilinear Calderón-Zygmund operators on Hardy spaces. Collect. Math. 52, 169-179 (2001)

15. Grafakos, L., Torres, R.H.: Multilinear Calderón-Zygmund theory. Adv. Math. 165(1), 124-164 (2002)

16. Huang, A.W., Xu, J.S.: Multilinear singular integral and commutators in variable exponent Lebesgue space. Appl. Math. J. Chin. Univ. Ser. B 25(1), 69-77 (2010)

17. Pérez, C., Torres, R.H.: Sharp maximal function estimates for multilinear singular integrals. Contemp. Math. $\mathbf{3 2 0}$ 323-331 (2003)

18. Xu, J.S.: Boundedness of multilinear singular integrals for non-doubling measures. J. Math. Anal. Appl. 327(1), 471-480 (2007)

19. Lu, Y., Zhu, Y.P.: Boundedness of multilinear Calderón-Zygmund singular operators on Morrey-Herz spaces with variable exponents. Acta Math. Sin. 30(7), 1180-1194 (2014)

20. Cruz-Uribe, D., Fiorenza, A., Neugebauer, C.J.: Weighted norm inequalities for the maximal operator on variable Lebesgue spaces. J. Math. Anal. Appl. 394(2), 744-760 (2012)

21. Muckenhoupt, B.: Weighted norm inequalities for the Hardy maximal function. Trans. Am. Math. Soc. 165, 207-226 (1972)

22. Cruz-Uribe, D., Diening, L., Hästö, P.: The maximal operator on weighted variable Lebesgue spaces. Fract. Calc. Appl. Anal. 14(3), 361-374 (2011)

23. Cruz-Uribe, D., Wang, L.A.: Extrapolation and weighted norm inequalities in the variable Lebesgue spaces. Trans. Am. Math. Soc. 369(2), 1205-1235 (2017)

24. Izuki, M.: Remarks on Muckenhoupt weights with variable exponent. J. Anal. Appl. 11(1), $27-41$ (2013)

25. Izuki, M., Nakai, E., Sawano, Y.: Wavelet characterization and modular inequalities for weighted Lebesgue spaces with variable exponent. Ann. Acad. Sci. Fenn., Math. 40(2), 551-571 (2015)

26. Li, X.W., Yang, D.C.: Boundedness of some sublinear operators on Herz spaces. III. J. Math. 40(3), 484-501 (1996)

27. Lu, S.Z., Yang, D.C., Hu, G.E.: Herz Type Spaces and Their Applications. Science Press, Beijing (2008)

28. Almeida, A., Hästö, P.: Besov spaces with variable smoothness and integrability. J. Funct. Anal. 258(5), 1628-1655 (2010)

29. Izuki, M., Noi, T.: Two weighted Herz spaces with variable exponents. Bull. Malays. Math. Sci. Soc. (2018). https://doi.org/10.1007/s40840-018-0671-4

30. Izuki, M., Noi, T.: Boundedness of fractional integrals on weighted Herz spaces with variable exponent. J. Inequal. Appl. 2016, Article ID 199 (2016)

31. Izuki, M., Noi, T.: An intrinsic square function on weighted Herz spaces with variable exponent. J. Math. Inequal. 11(3), 799-816 (2016)

32. Sawano, Y.: Theory of Besov Spaces. Springer, Singapore (2018)

\section{Submit your manuscript to a SpringerOpen ${ }^{\circ}$ journal and benefit from:}

- Convenient online submission

- Rigorous peer review

- Open access: articles freely available online

- High visibility within the field

- Retaining the copyright to your article

Submit your next manuscript at $\gg$ springeropen.com 